\title{
Dynamical Approach for Real-Time Monitoring of Agricultural Crops
}

\author{
Fernando Vicente-Guijalba, Tomas Martinez-Marin, and Juan M. Lopez-Sanchez, Senior Member, IEEE
}

\begin{abstract}
In this study a novel approach for exploiting multitemporal remote sensing data focused on real-time monitoring of agricultural crops is presented. The methodology is defined in a dynamical system context using state-space techniques, which enables the possibility to merge past temporal information with an update for each new acquisition. The dynamic system context allows us to exploit classical tools in this domain to perform the estimation of relevant variables. A general methodology is proposed, and a particular instance is defined in this study based on polarimetric radar data to track the phenological stages of a set of crops. A model generation from empirical data through a principal component analysis is presented, and an extended Kalman filter is adapted to perform the phenological stage estimation. Results employing quad-pol Radarsat- 2 data over three different cereals are analyzed. The potential of this methodology to retrieve vegetation variables in real time is shown.
\end{abstract}

\section{Keywords}

Dynamic system, state space, real-time, multitemporal, Kalman, SAR, agriculture, phenology, polarimetry.

\section{INTRODUCTION}

D URING the last decades remote sensing has been an extremely important source of information to improve the human knowledge about the dynamical process occurring in our planet. In particular, data from both optical sensors [1], [2], [3] and radar systems [4], [5], [6] have been applied to agricultural crop monitoring. In this context, timely and spatially fine information about the condition of crops is an increasing demand from both farmers (to help precision farming methods) and institutions at different spatial levels (to improve yield forecasts or to ensure environment-friendly practices). Farming management by growth stage is critical to optimize returns from inputs such as nitrogen (N), plant growth regulator (PGR), fungicides and water. For instance, the key growth stages for fungicide application are often within the stem elongation stage, and any variation in the application timing can affect the final yield [7]. To provide such a frequent update of information, optical data [8], [9] have an inherent limitation when cloud cover is present. Alternatively, synthetic aperture radars (SAR) can be used, provided their night-and-day operation and near all-weather independence. In addition, current and upcoming SAR missions (e.g. TerraSAR-X, Radarsat-2, Sentinel-1, Radarsat Constellation Mission) have been designed to increase their revisit time, achieving very low values (e.g. 6 days with the pair of Sentinel-1 satellites) as required by this sort of application. However, the processing of time series of remote sensing data for monitoring the development of agricultural crops is not mature. In this paper we propose a general framework to exploit jointly the information provided by each acquisition and the expected evolution of the crops with the aim of producing better estimates of the phenological stage of the observed crops.

Classical approaches employ a single or a subset of pixels from the original signal (in either temporal or spatial domain) with the aim to enhance a particular value or to infer an external variable. This is the situation, for instance, in a smoothing algorithm which employs a pixel's neighborhood to compute its smoothed value. The original observed values are the only source of information used to compute the filtered data. Dealing with a biological process, however, there exist regularities in the growing cycle which limit the evolution of some parameters between acquisitions. Thus, the inclusion of any knowledge of the dynamics of the involved process in the estimation or filtering algorithm should improve the final results.

In this study, the phenological process of a crop, i.e. its growth or development, is interpreted as a dynamical evolution which responds to an unknown analytical model that depends on a huge number of variables, such as temperature, humidity, irrigation, soil conditions, solar irradiance, etc. A methodology is proposed to extract the evolution model of a generic crop, either for the phenology or any other biological process, in a first stage. The final objective is to exploit the created model to estimate, in a second stage, the growth stage in which a particular crop field is. In order to develop the potential of remote sensing acquisitions, the estimation step is carried out in real-time. This means that as soon as new input data are available (e.g. a new satellite image is acquired), the estimation is applied, so it is not necessary to perform the estimation over the whole temporal series.

The definition of both learning and estimation stages are derived in the state-space context. Besides the application of well known tools of dynamic system theory, this context allows us to describe completely the system at each moment with a single state. This idea was firstly introduced in [10]. Dual-pol SAR data were used as input and the estimation of the phenology of rice fields was obtained using a Extended Kalman Filter (EKF). It is important to mention that different approaches have been defined

\footnotetext{
This work is supported by the Spanish Ministry of Economy and Competitiveness (MINECO) and EU FEDER under Project TEC2011-28201-C02-02, and by the Generalitat Valenciana under Project ACOMP/2014/136.

The authors are with the Institute for Computing Research (IUII), University of Alicante, P.O. Box 99, E-03080 Alicante, Spain (e-mail: fernando.vicente@ ua.es; tomas@dfists.ua.es; juanma-lopez@ieee.org)
} 
in the literature using a similar EKF estimation approach. In [11] a soil moisture estimation is proposed based on SAR data, and in [12] a land cover change using NDVI MODIS data is analyzed similarly. In both cases, the application of the EKF estimator relies directly upon a known analytical model, being the main difference with the methodology introduced in this framework.

With respect to [10], a deeper analysis of the methodology and a more complete evaluation over different cereal crops is carried out in this work. Although in this study we exploit a temporal stack of SAR images, the methodology is not limited to this data type, since the same approach is valid for other remote sensing data (e.g. optical or NDVI products) or even in a combined methodology.

Finally, there exists the option to work with an approach based on single acquisitions to estimate the phenological stage. For instance, in [13], [14] independent SAR acquisitions were employed for the estimation of phenology over rice fields. That approach will be named hereafter as statical in contrast to the dynamical framework proposed in this paper. Nonetheless, temporal series of images were also analyzed as part of the training stage to define the employed thresholds. It was shown that with such an approach rice field phenology was traceable with classification trees employing large phenological intervals, i.e. a wide discretization of the original scale. The main drawback of this sort of techniques, apart from the wide phenological classes employed, is that it is necessary to study beforehand the behavior of all polarimetric observables to determine which of them are useful to perform the estimation. In addition, being based on an empirical analysis, it is not guaranteed that the chosen thresholds are convenient for other datasets. Clear improvements from this statical approach are the provision of an automatic analysis and a finer resolution in the estimation of the phenology, which is a key aspect from the end users' point of view. Recent studies have also proved the potential of different SAR products in agricultural monitoring, as in [15] where interferometric bistatic products are employed to derive biophysical parameters in rice fields.

The paper is organized as follows. The tools and methodologies employed in this study are introduced in Section II. The definition of the dynamical context and the general approach for real-time monitoring are stated. The particular approach employed in this study is also described. The dataset and the results obtained with this methodology over different cereal crop types are shown and discussed in Section III. Finally, conclusions are exposed in Section IV, together with some ideas for future work.

\section{MATERIALS AND METHODS}

The theoretical aspects related to the presented methodology are all described in this Section. First, a brief introduction to dynamic systems is provided in Section II-A with the definition of the classical Kalman filter approach. A general view of the methodology is also provided. In the three subsequent points, the particular instance of this methodology, employed in this work, is described. This includes the state-space description based on polarimetric data in Section II-B, the evolution model generation in Section II-C, and the details of the filtering strategy in Section II-D.

\section{A. Dynamic approach}

A system that evolves in time according to a rule is known as a dynamic system. The temporal evolution of such a system is defined as a process. The rules of evolution are commonly governed by the following pair of equations [16], [17], [18]:

$$
\begin{aligned}
\dot{\mathbf{x}}(t) & =\frac{d \mathbf{x}(t)}{d t}=\mathbf{f}(\mathbf{x}(t), t, \mathbf{v}(t)) \\
\mathbf{z}(t) & =\mathbf{h}(\mathbf{x}(t), t, \mathbf{w}(t))
\end{aligned}
$$

where Eq. (1) represents the recursive process equation, describing the evolution of the system over time, and Eq. (2) defines the measurement equation, describing the observation relation with the system. The $n$-dimensional state vector or, simply, state $\mathrm{x}(t)$ collects into a single vector the set of $n$ state variables $x_{i}$ which completely describe the system configuration at time $t$. The $n$-dimensional domain in which the state is defined is called the state space. The set of functions providing the rate of change of the state for a particular stage and time are represented by $\mathbf{f}()$. The noise in the evolution is modeled by the stochastic process $\mathbf{v}(t)$. In the measurement equation, the measurement or output vector $\mathbf{z}(t)$ is defined by the measurement function $\mathbf{h}()$ which relates the internal state vector with the observed $\mathbf{z}(t)$. The observation process is also affected by a noise process $\mathbf{w}(t)$.

One of the most remarkable features of the dynamic system approach in the state-space domain is that the information related to the past of the process is merged into the current state. This is usually known as a Markovian process. In this scenario, once the system is already defined, a prediction of a state $\mathbf{x}(t)$ at any time can be obtained based only on the system state $\mathbf{x}\left(t_{0}\right)$ for a particular time $t_{0}<t$ [16]. Moreover, this state is recursively updated as data arrive, thus making the state-space modeling a proper approach to process data with temporal dependencies.

The dynamic system introduced in Eqs. (1) and (2) represents a continuous system because it is defined with respect to the continuous independent variable $t$. However, for many practical problems, the interest relies only in some states at a discrete set of times. For this type of problems it is convenient to sort the times according to an integer subscript $t_{0}<t_{1}<t_{2}<\cdots<t_{k-1}<t_{k}$, redefining the state of the dynamic system as in Eq. (3):

$$
\mathbf{x}\left(t_{k}\right)=\mathbf{f}\left(\mathbf{x}\left(t_{k-1}\right), t_{k-1}, \mathbf{v}\left(t_{k-1}\right)\right)
$$


where the set of equations $\mathbf{f}()$ is transformed from the continuous domain (derivatives) to the discrete domain (difference equations). Note that the $\mathbf{f}()$ functions that appear in (1) and (3) are completely different. However, we have preferred to keep the same notation in both equations as it is usually done in the literature. In addition, it is more efficient to shorten the notation, so, as long as it is understood $\mathbf{x}\left(t_{k}\right)$ will be expressed as $\mathbf{x}_{k}$. Finally, under an additive noise assumption, the general expression for a dynamic system in the estimation framework is expressed by Eqs. (4) and (5):

$$
\begin{aligned}
\mathbf{x}_{k} & =\mathbf{f}_{k-1}\left(\mathbf{x}_{k-1}\right)+\mathbf{v}_{k-1} \\
\mathbf{z}_{k} & =\mathbf{h}_{k}\left(\mathbf{x}_{k}\right)+\mathbf{w}_{k}
\end{aligned}
$$

It has to be noted that the additive noise corresponds to the process noise or uncertainty in the state space, and not the noise present directly in the values of the input data (e.g. speckle in SAR images).

Some considerations are also usually adopted in practice, being the most usual to consider: a) a linear system, and b) process and measurement noises both additive white Gaussian processes. One of the most known approaches under these conditions is the Kalman filter (KF) [19], [20]. The KF was proposed in order to obtain optimal estimations for linear systems under Gaussian hypotheses, both in the transition and in the observation. Later on, different techniques were developed in order to handle nonlinear dynamic systems with the same general approach, as the Extended Kalman filter (EKF).

This method allows to get a simple approximation to the solution for a nonlinear estimation problem using the linear approximation described by Eqs. (6) and (7):

$$
\begin{aligned}
J_{k-1} & \left.\approx \frac{\delta \mathbf{f}_{k}}{\delta \mathbf{x}}\right|_{x=\hat{x}_{k-1}} \\
H_{k} & \left.\approx \frac{\delta \mathbf{h}_{k}}{\delta \mathbf{x}}\right|_{x=\hat{x}_{k}}
\end{aligned}
$$

where $J_{k-1}$ corresponds to the linearized transition matrix at state $k-1$, and $H_{k}$ is the linearized measurement matrix at state $k$. It is noted that the linearization is particularized over the estimated state vector $\hat{x}_{k-1}$. Exploiting these approximations the nonlinear problem can be described in the same terms as the classical KF.

The estimation algorithm is described in two main stages, namely prediction and update. In the prediction stage, based on the transition model and the previous state, an a priori estimation for the state and the covariance is provided. As soon as an observation is available, the algorithm is able to provide an innovation, as the difference between the observed value and the predicted one. The update stage is driven by the Kalman gain, which provides the optimal (suboptimal for EKF) weighting for the predicted and the innovation states. As a result, after the update stage, an a posteriori state and a covariance matrix are generated. It is important to note that the update is done when an observation is available, and there is no need to hold it on for future observations, hence defining a real-time or online estimation procedure. For the sake of clarity, the stages just described, corresponding to the EKF approach, are summarized in Table I.

In our application, the development of a crop field, i.e. its phenology, can be considered as a process of a particular dynamic system. For this reason, in this work we propose this methodology, focused on exploiting the potential provided by these filtering strategies, such as the EKF. With this objective, a conceptual implementation of the whole methodology is shown in Fig. 1. The functional model consists of two main blocks, specifically the model generation and the estimation stage. The aims of the first one are to assess the state-space domain of the system and to generate the dynamic process evolution in the defined state space. This block can be interpreted as a learning stage from a reference set of data. The second block is devoted to, exploiting the generated dynamic process, estimate the external variable for a set of input data through a dynamic filter approach. Although this estimation employs the evolution model, the estimation is performed online (or in real-time) whenever a new observation is incorporated to the process.

Besides the functional model, the approach is defined by the data. First, the reference dataset, which is used to extract the model evolution, is defined by different temporal acquisitions $\left(t_{i}\right)$ sampling the relevant temporal range. Each acquisition is described by a characteristics vector $\left(\mathbf{u}_{i}\right)$ and the associated crop variable $\left(Y_{i}\right)$. The characteristics vector is related with the remote sensed values, whereas the crop variable defines the biological process we are interested to track or estimate. Second, the input dataset represents the temporal acquisitions, with their associated characteristics vector, that are evaluated through the estimation algorithm. Each acquisition is projected into the defined state space and employed to provide an estimation of the crop variable (e.g. phenological stage) which defines the output data of the algorithm. As depicted in the figure, an optional feedback can be finally exploited to regenerate or refine the evolution model after each estimation is obtained, hence providing an online modeling for the model generation.

In the approach described in this work, the dates of sowing of the crop fields in the reference dataset and in the area to be monitored are required. However, this in situ information is usually not available in the fields in which phenological estimates are to be obtained. To solve this issue, and also as a standalone application of this general methodology, the model can be also 
TABLE I. ESTIMATION STAGES AND DEFINITIONS IN EKF

\begin{tabular}{cl}
\hline Stage & \multicolumn{1}{c}{$\hat{\mathbf{x}}_{k \mid k-1}=\mathbf{f}_{k-1}\left(\hat{\mathbf{x}}_{k-1 \mid k-1}\right)$} \\
\hline \hline Prediction & $P_{k \mid k-1}=J_{k-1} P_{k-1 \mid k-1} J_{k-1}^{T}+Q_{k-1}$ \\
\hline Innovation & \multicolumn{1}{c}{$\tilde{\mathbf{y}}_{k}=\mathbf{z}_{k}-\mathbf{h}_{k}\left(\hat{\mathbf{x}}_{k \mid k-1}\right)$} \\
\hline Kalman Gain & $K_{k}=P_{k \mid k-1} H_{k}\left(H_{k} P_{k \mid k-1} H_{k}^{T}+R_{k}\right)^{-1}$ \\
\hline Update & \multicolumn{1}{c}{$\hat{\mathbf{x}}_{k \mid k}=\hat{\mathbf{x}}_{k \mid k-1}+K_{k} \tilde{\mathbf{y}}_{k}$} \\
& $P_{k \mid k}=\left(I-K_{k} H_{k}\right) P_{k \mid k-1}$ \\
\hline \hline Definitions & \\
\hline$\hat{\mathbf{x}}_{i \mid j}$, & state vector estimation at $i$ given $j ;$ \\
$\mathbf{f}_{i}()$, & transition model at $i ;$ \\
$J_{i}$, & linearized transition matrix at $i ;$ \\
$Q_{i}$, & model covariance at $i ;$ \\
$P_{i \mid j}$, & prediction covariance at $i$ given $j ;$ \\
$\mathbf{z}_{i}$, & data observation at $i ;$ \\
$\mathbf{h}_{i}()$, & measurement model at $i ;$ \\
$\tilde{\mathbf{y}}_{i}$, & innovation at $i ;$ \\
$H_{i}$, & linearized measurement matrix at $i ;$ \\
$K_{i}$, & Kalman gain at $i ;$ \\
$R_{i}$, & observation covariance at $i$.
\end{tabular}

exploited backwards in time to provide estimates of the date of sowing and other important dates for the farming practices. This variant of the methodology is described and validated in [21].

In order to obtain an operational approach, a specific instance of the methodology is defined. Different strategies can be applied over the different sub-blocks of the methodology. For instance, the input data can be derived from different remote sensing systems, such as optical, hyperspectral or SAR, or even a combination of them. For the case of the estimated output variables, alternatives as the phenological stage or the leaf area index (LAI) can be considered. Regarding the functional model, also different options can be taken into account. In the case of the learning strategy, algorithms based on principal component analysis (PCA), support vector machines (SVM) or artificial neural networks (ANN) can be considered. It has to be remarked also the importance of the employed reference dataset (i.e. the training set). The employed temporal set should be representative enough of the space of occurrences, allowing the learning stage to build a general model about this space that enables it to produce sufficiently accurate predictions for new scenarios. Moreover, there are different approximations based on filtering for the estimation approach, such as the EKF, the grid-based filter or the particle filter, all of which fit into the described methodology. As it is described in the following, in this study a implementation based on an input polarimetric dataset is considered to estimate the phenological stage for different cereal crop types.

\section{B. PolSAR State-Space definition}

Fully polarimetric observations of distributed scenes in a monostatic configuration, assuming reciprocity, are defined by their multi-looked covariance matrix $\mathrm{C}$ as in Eq. (8):

$$
\begin{aligned}
C & =\frac{1}{N} \sum\left\langle\mathbf{k k}^{* T}\right\rangle \\
& =\left[\begin{array}{ccc}
\left\langle|H H|^{2}\right\rangle & \sqrt{2}\left\langle H H H V^{*}\right\rangle & \left\langle H H V V^{*}\right\rangle \\
\sqrt{2}\left\langle H V H H^{*}\right\rangle & 2\left\langle|H V|^{2}\right\rangle & \sqrt{2}\left\langle H V V V^{*}\right\rangle \\
\left\langle V V H H^{*}\right\rangle & \sqrt{2}\left\langle V V H V^{*}\right\rangle & \left\langle|V V|^{2}\right\rangle
\end{array}\right]
\end{aligned}
$$

where $\mathbf{k}=\left[\begin{array}{lll}H H & \sqrt{2} H V & V V\end{array}\right]^{T}$ is the polarimetric scattering target vector, and the operator \langle\rangle represent the multi-look process over $N$ independent looks. It is also common to use the Pauli basis to represent the data, taking the form of the polarimetric coherency matrix $\mathrm{T}$, which can be obtained through a unitary transformation from $\mathrm{C}$ (both are Hermitian positive semidefinite matrices) [22], [23]. The set of input parameters (observables) employed in this study is obtained from both matrices, and initially can be divided into two main groups: power of different channels (diagonal terms), and complex correlations between channels (off diagonal terms) providing each one a normalized correlation and a phase difference between channels. In addition, from both matrices different decomposition techniques, aimed to provide a physical interpretation, can be applied. In this work the set of 


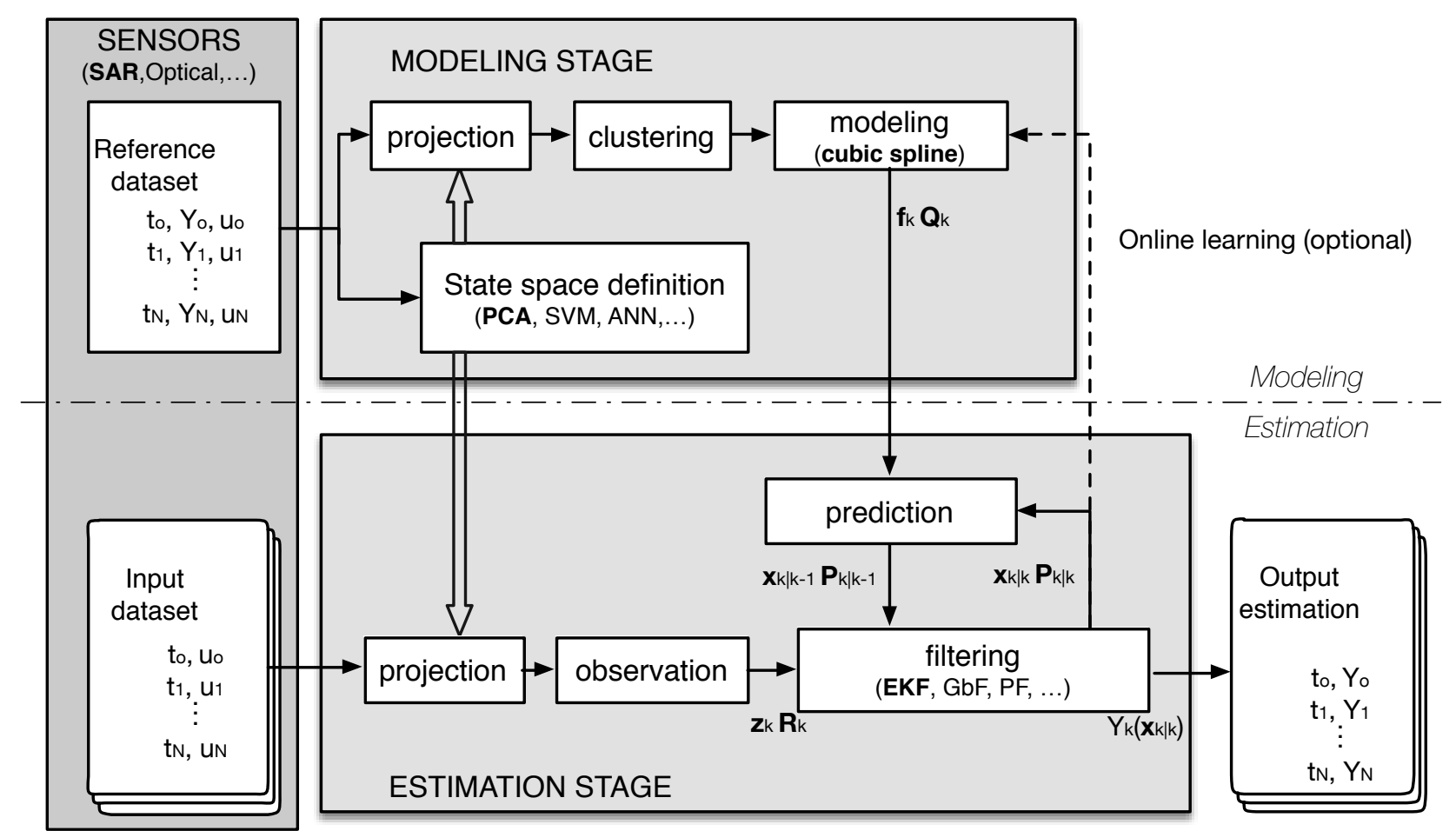

Fig. 1. Block diagram of the dynamic approach for agricultural crop monitoring. The approach is divided in two functional blocks: model generation and estimation stage. A reference dataset is employed to extract the dynamical model. An input dataset is used for estimation.

observables is complemented with the $H / A / \alpha$ polarimetric parameters introduced in [24]. Using this reference, each observation is described as a set of sixteen different parameters: 5 power terms, 4 normalized correlation values, 4 phase differences, and 3 parameters from the eigenvector decomposition of matrix T. It has to be reminded that this selection of initial parameters is just an instance of the methodology. Indeed, a complete analysis of the performance as a function of the input domain could be carried out and would be subject of further discussion, but it is beyond the scope of this manuscript. It should be noted that this scheme is also suitable for different polarimetric configurations (e.g. dual-pol, hybrid or circular polarizations), and this will be analyzed in a future study.

In order to define a state space with easy representation, for illustration purposes, in this study the state vectors $\mathbf{x}_{k}$ are derived from the proposed set of 16 observables after a dimensional reduction. A principal component analysis (PCA) is employed, whose main goal is to identify the most meaningful basis to re-express the original polarimetric domain. Therefore, the idea is to employ each polarimetric feature evolution as input to the analysis. The input data consists in the whole set of all merged reference parcels, described by their polarimetric parameters, to define the common output space, which is later used to project each parcel evolution. Let $\mathbf{Y}_{\mathbf{m x n}}$ be the original polarimetric data of $m$ observables and $n$ samples (to which a standardize operation has been applied) for each parcel and date. The PCA approach allows us to obtain a new basis $\mathbf{X}_{\mathbf{m} \times \mathbf{n}}$ which is related to the original one by a linear transformation $\mathbf{A}_{\mathbf{m x m}}$. Each row of the transformation matrix corresponds to a principal component and represents the set of new basis vectors used for expressing the columns of $\mathbf{Y}$. As the principal components are sorted in terms of variance, the dimensional reduction in this domain consists in a truncated transformation matrix. As we will show in Section III, from the original dataset $(m=16)$ it is sufficient to consider only the first three components, thus $\mathbf{A}_{\mathbf{3 \times n}}$. With this consideration, variance is held at $70 \%$ of the original value, and the interpretation of the state space can be derived geometrically. In this way the projected domain over the three main principal components defines the state $\mathbf{x}_{k}$ of each parcel observation.

\section{Evolution model}

A classical dynamic approach is defined using an analytical function characterizing both the evolution rules $\mathbf{f}_{k-1}()$ and the system output $\mathbf{h}_{k}()$. In our case, due to the nonexistence and complexity of such an analytical model, the dynamics is extracted 
directly from the observed data. This learning stage produces a representative model evolution for the kind of crops under study.

For the reference set of parcels, the dynamical evolution is obtained in two steps. The first one involves the computation of the polarimetric observables at parcel level, i.e. only one covariance matrix is computed for a whole parcel at each temporal acquisition, and the projection into the space provided by the PCA. The extraction of the principal components had to be carried out previously as described in Section II-B. Each acquisition is projected to the state space defined by the dimensions expressed as linear combinations of the polarimetric information as in Eq. (9):

$$
x_{n}=\sum_{i=1}^{m} a_{i} p_{i} \quad \text { for } n=0,1, \ldots, m-1
$$

where $a_{i}$ denotes the coefficient (provided by the PCA for each dimension) associated with each polarimetric observable $p_{i}$, and $x_{n}$ is the projected value in the $n$-th dimension. The number of the original parameters $m$ limits the dimension of the projected space. So far, each field at each date is represented by a coordinate vector $\left[x_{0} x_{1} \ldots x_{m-1}\right]$ in the projected space. In order to illustrate this, Fig. 3 shows the projection over the two first components obtained for each acquisition at three different barley fields using a stack of 24 images. In the state plane defined by the first two components $\left(x_{0}\right.$ and $\left.x_{1}\right)$ each field data at each acquisition is represented by an up/down triangle for ascending/descending tracks, and the color code denotes the beam (incidence angle) for each case. Moreover, each of them has an associated phenological interval, minimum and maximum, provided by the ground campaign. The available phenological information corresponds to the scale defined in [25], coincident with the standard $\mathrm{BBCH}$ scale [26], ranging from 0 to 100 in a continuous way. A scheme of the scale and their main stages is shown in Fig. 2.

\begin{tabular}{|c|c|c|}
\hline & ale & \\
\hline 10 & Germination & \\
\hline & Leaf development & \\
\hline & Tillering & Vegetative \\
\hline & Stem elongation & \\
\hline & Booting & \\
\hline & Infloresc. emergence & odu \\
\hline & Flowering & IIV \\
\hline & Fruit development & \\
\hline & Ripening & Maturation \\
\hline & Senescence & \\
\hline
\end{tabular}

Fig. 2. Phenological scale employed in the ground campaign. The scale provides a continuous representation of the crop evolution.

The absence of a continuous phenological record, however, prevents us to generate directly a model describing the whole set of phenological values in the $\mathrm{BBCH}$ scale. Instead, a discretization from the original continuous range is performed to provide a reasonable sampling in the evolution model. Thus, the model is identified in phenological discrete intervals or clusters which enclose neighbor ranges of phenological values.

The second step consists in obtaining the evolution model in the projected space. A geometrical model is defined for a predefined set of phenological clusters given by the phenological ranges they represent. Every acquisition falling inside a phenological range is employed to obtain the center of each cluster, $\mathbf{x}_{k}$, and the associated covariance matrix, $Q_{k}$. For instance, in Fig. 3 the creation of the first cluster, $x_{k}=0$, is illustrated. In this case there are 6 elements (the ones linked with dotted lines to the cluster center) in the plane with $\mathrm{BBCH}$ values in the range $[0-12]$ and they are employed to compute the mean and the covariance value. This clustering is done for each specified phenological interval. After that, the model is defined by $\mathbf{x}_{k}, Q_{k}$ and the phenological range $B B C H_{k}$ at $k=0,1,2, \ldots, K-1$, where $K$ corresponds to the number of clusters predefined as input. To complete the model definition, the local temporal variation $\delta \mathbf{f}_{\mathbf{k}}=\delta \mathbf{x}_{\mathbf{n}} / \delta t$ of each cluster is also computed from the state vector variation and the temporal acquisition ranges of each cluster. As a result, the velocity state vector is also provided at each cluster.

In Fig. 3 a total of $K=9$ clusters are represented on this bidimensional state space, and the variance at each dimension (i.e. diagonal elements of the covariance matrix) is denoted by an ellipse. The continuous evolution, represented by the solid black line connecting the different cluster centers, is obtained by means of a cubic splines interpolation.

\section{Phenological Estimation Approach}

At this stage, most of the estimation techniques are usually focused on exploiting either the model or the observations, but not both. In the first case, the introduced geometrical model is assumed to describe fully the crop signature, despite it may be limited by the set of parcels employed in its generation. If the fields employed in the model generation are representative enough, the model can be used directly to describe how any field behaves or evolves in time using exclusively temporal information over the model. In that case, state vectors would be used to provide transitions from one cluster to the next, allowing also the access to intermediate interpolated values. With such a methodology, the phenological value for each estimation would be retrieved directly from the cluster assigned after each temporal transition. In the second case, which corresponds to a classical filtering 


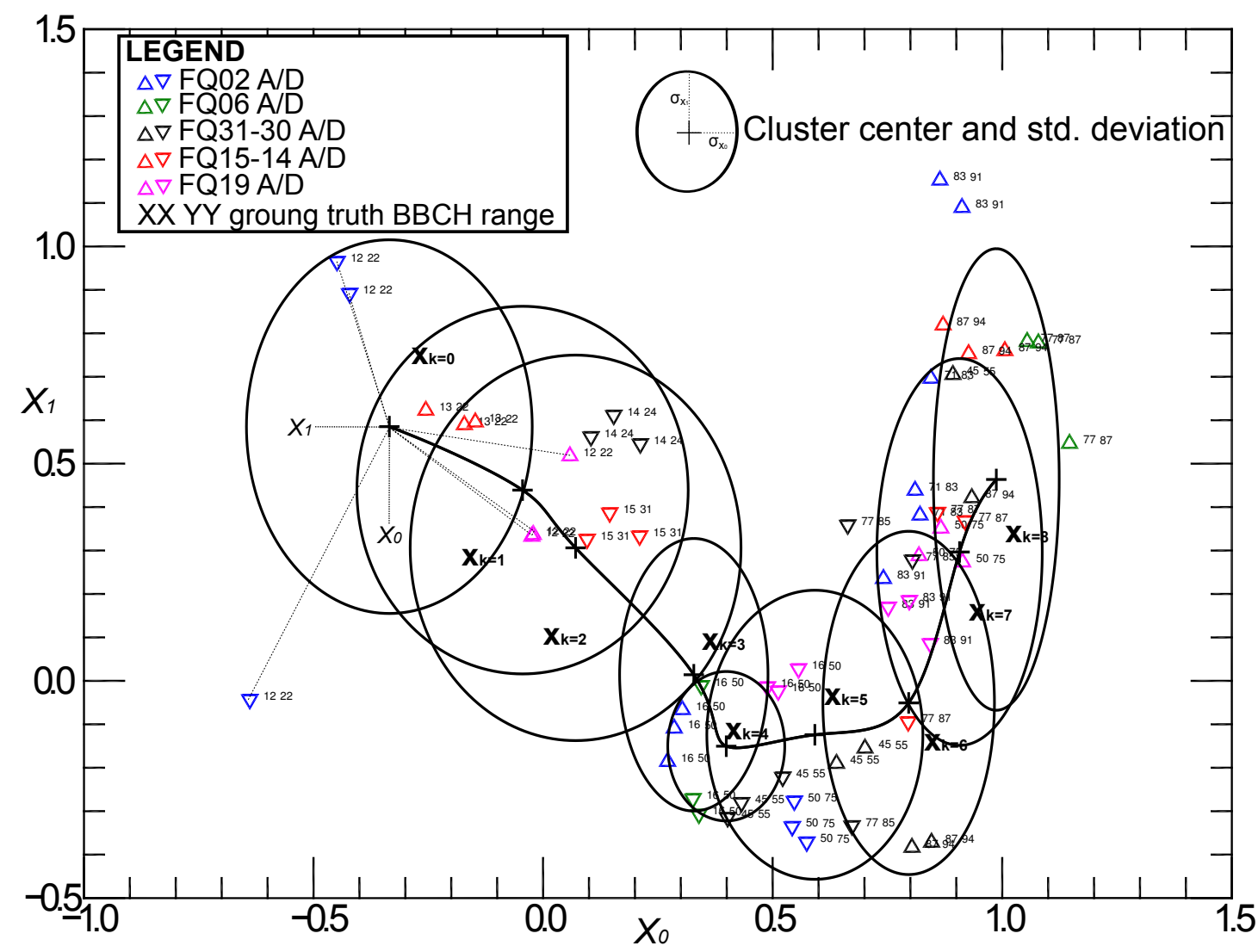

Fig. 3. Two-dimensional projection of the reduced space for all Radarsat-2 beams and tracks using three barley crops. Each triangle represents a field acquisition and the subscripts denote the ground truth provided for the phenological stage at the acquisition date. A total of $\mathrm{K}=9$ phenological intervals are employed in the clustering process. Results are presented by the cross-marks and the standard deviation of each component is directly represented by an ellipse. The transition between states described by the continuos line is obtained trough a cubic spline interpolation.

approach, the observation could be filtered with a fixed rule (or function) without incorporating any other knowledge to the process. In both cases there would be a bias from the real evolution, in contrast with the results that can be obtained by properly combining both sources of information: model and observations.

In order to combine the information of the previously obtained model and that provided by the observations, a filtering strategy based in EKF is proposed in the methodology. The most common way to work with this dynamic approach is using an analytical transition and measurement functions. Nevertheless, it is possible to obtain the functions that define the dynamic of the process in alternative ways to the analytical case. In this work, we propose to bypass the absence of an analytical model by using a numerical evolution obtained directly from the empirical observations. The required linearization is not based directly on the analytical Jacobian matrix. Instead, a numerical derivation using the model is performed. The linearized approximation to the state transition matrix in the state $k-1$ is expressed as in Eq. (10):

$$
J_{k-1}=\left[\begin{array}{lll}
\frac{\delta f_{k}^{0}-\delta f_{k-1}^{0}}{x_{k}^{0}-x_{k-1}^{0}} & \frac{\delta f_{k}^{0}-\delta f_{k-1}^{0}}{x_{k}^{1}-x_{k-1}^{1}} & \frac{\delta f_{k}^{0}-\delta f_{k-1}^{0}}{x_{k}^{2}-x_{k-1}^{2}} \\
\frac{\delta f_{k}^{1}-\delta f_{k-1}^{1}}{x_{k}^{0}-x_{k-1}^{0}} & \frac{\delta f_{k}^{1}-\delta f_{k-1}^{1}}{x_{k}^{1}-x_{k-1}^{1}} & \frac{\delta f_{k}^{1}-\delta f_{k-1}^{1}}{x_{k}^{2}-x_{k-1}^{2}} \\
\frac{\delta f_{k}^{2}-\delta f_{k-1}^{2}}{x_{k}^{0}-x_{k-1}^{0}} & \frac{\delta f_{k}^{2}-\delta f_{k-1}^{2}}{x_{k}^{1}-x_{k-1}^{1}} & \frac{\delta f_{k}^{2}-\delta f_{k-1}^{2}}{x_{k}^{2}-x_{k-1}^{2}}
\end{array}\right]
$$

where each differential coefficient is understood as an approximation to the partial derivatives from the classical Jacobian matrix, but each value is obtained directly from the model. In this methodology, the observation matrix $H_{k}$ is considered as an identity matrix provided that the observations $\mathbf{z}_{k}$ are already provided in the projected state space using Eq. (9). The covariance matrix $R_{k}$ for each observation is computed through a Monte Carlo simulation for PolSAR data as described in [22], [23]. Speckle noise is simulated for each observed polarimetric covariance matrix $C$ and used to compute, through the linear combination of Eq. (9), a representative distribution of the observed state. From this distribution the covariance matrix $R_{k}$ of the observation 
in the state space is computed. The Monte Carlo simulation is evaluated over each multi-looked $C$ matrix under the estimation stage. The purpose is to create a set of instances of the process behind each observation under estimation. Then the instances are employed to extract the covariance matrices of the polarimetric parameters and hereafter the covariance of the PCA variables. The number of samples employed in each simulation is $N=1000$.

Extending the basis of the EKF algorithm, the phenological stage retrieval is divided into two main stages: prediction and update. The prediction stage makes use of the model evolution and the transition matrix. A numerical integration in time is employed to compute an a priori estimate for the state $\hat{\mathbf{x}}_{k \mid k-1}$ and the associated covariance matrix $P_{k \mid k-1}$, using just the previous state estimate $\hat{\mathbf{x}}_{k-1 \mid k-1}$. It is important to emphasize that this a priori estimation is based only on the previous state and not in earlier ones.

The second stage (update) occurs when a new data observation is available (e.g. a new satellite image is acquired). The objective of this second stage is to improve, using sensed data, the previously predicted value by minimizing the covariance of the estimation. The required input is composed by two parts: the acquired polarimetric observables projected in the state space, $\mathbf{z}_{k}$, and their associated covariance matrix, $R_{k}$. The innovation product $\hat{\mathbf{y}}_{k}$ and the Kalman gain are obtained using the equations defined in the EKF algorithm (Table I), giving a measure of the improvement with respect to the prediction and the observed data. Both parameters are employed to compute the a posteriori state, $\hat{\mathbf{x}}_{k \mid k}$ and its covariance matrix $P_{k \mid k}$, providing an updated and final state estimate for the current acquisition. Finally, to supply a phenological value in the BBCH scale, the estimated state is projected to the original evolution model, which bears the corresponding phenological values.

\section{RESULTS}

Some first results employing the presented methodology were shown in [10]. A stack of dual copolar TerraSAR-X images was employed over rice fields to check the methodology effectiveness. These results showed a clear improvement with respect to the static results presented in [13], although a quantitative assessment was not carried out. However, static methodologies have proven to be effective in the phenological estimation problem over different types of crops using SAR data. In [27] it is shown that a very high accuracy in the phenological estimation is reached for some crop types. Nevertheless, the way to export this static methodology to different scenarios is not well defined yet. In contrast, the design of the multitemporal approach proposed here has been defined specifically for this purpose.

In this Section, an example of the practical operation of the presented methodology over a set of cereal fields is presented, together with an analysis of the obtained results. The input dataset and the localization of the fields under study are introduced in Section III-A. The pre-processing of the polarimetric data, required before this methodology is applied, is described in Section III-B. Details regarding the state-space definition and the model generation are discussed in Section III-C. Finally, the obtained estimates are shown and analyzed in Section III-D.

\section{A. Datasets}

In order to test the presented methodology, we employed the data provided by the ESA-funded AgriSAR 2009 campaign over Indian Head (Saskatchewan, Canada) where different crop fields were intensively monitored both with remote sensors and on the ground [28]. The precise location of the area under study is shown in Fig. 4. Three different cereal crops are considered in this work: barley, wheat and oat.

The available phenological information at the monitored fields is represented graphically in Fig. 5. The ground measurements were carried out in a weekly basis (every 7 or 10 days). At each date, an interval of values (lowest and highest phenological values present in a particular field) is provided (denoted by cross marks in Fig. 5). Since the dates of acquisition of the ground data are not coincident with the SAR acquisitions, a sort of interpolation is required. Attending to the expected behavior of the agricultural crops, the region of possible values is extended in the following way: each minimum value defines the lower limit until a new minimum value is specified, and each maximum value can be present from the previous date until it is measured. As a result, the possible phenological values as a function of date exhibit the staggered aspect shown in the figure.

The original set of SAR data consists of 57 Radarsat-2 images, all in fine quad-pol mode, with incidence angles ranging from $22^{\circ}$ to $39^{\circ}$ in both ascending and descending orbits. From the whole set, only the images with phenological information (i.e. from sowing to harvest) have been considered, covering the 3-month period between June 1 and August 31, and no restriction related to weather conditions has been contemplated. Therefore, the analysis is restricted to 24 valid images from all beams and incidence angles, listed in Table II.

The influence of including very different incidence angles in the model generation is an issue to be carefully analyzed. Some polarimetric observables are more influenced than others by incidence angle, so a selection of them could be a good strategy to avoid mixing different behaviors. This may be especially important when extreme angles are incorporated evenly to the model (like 22 and 39 degrees as in our case). Another possible approach would consist in restricting the dataset to only similar angles, hence avoiding this issue. However, in order to provide a valuable tool in the dynamical framework, it is highly important to hold the shortest sampling rate. Although some differences in extreme incidence angles are detected, we decided to exploit here as long as possible the similarities in the common behavior. In fact, if we try to reduce the number of images (i.e. the number of incidence angles) it is not possible to know in this scenario whether the benefits of not mixing incidence angles 


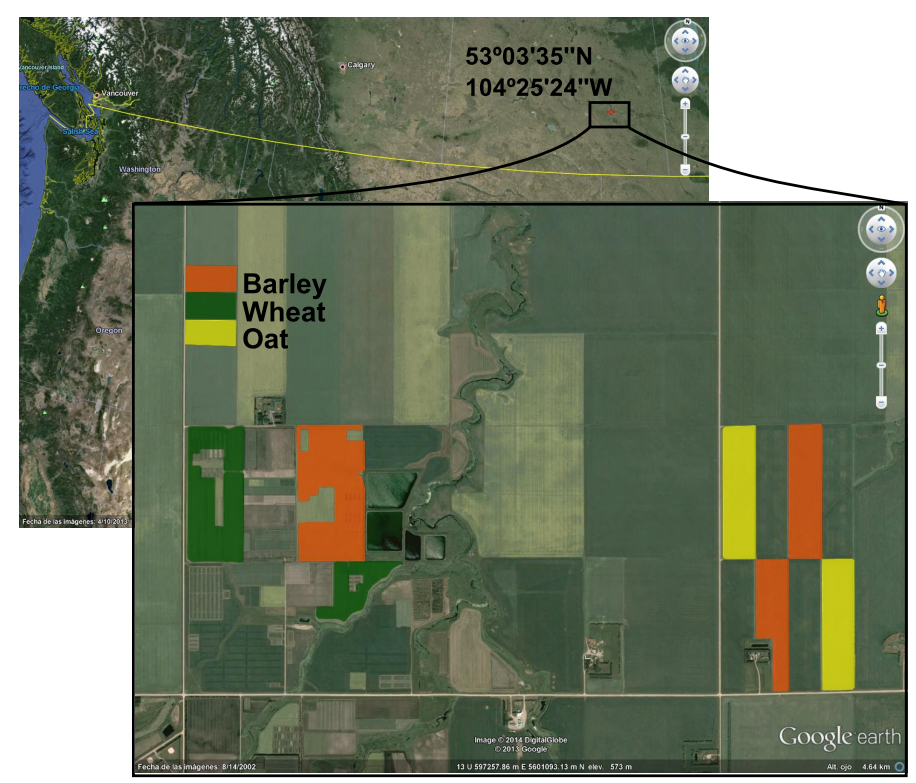

Fig. 4. Crop fields localization and distribution in the analyzed area. A total of seven fields are employed: three barley fields (orange), two wheat fields (green) and two oat fields (yellow). Photos courtesy of Google Earth.

TABLE II. LIST OF ACQUISITIONS EMPLOYED IN THE ANALYSIS.

\begin{tabular}{ccccc} 
Date (yyyymmdd) & DoY & Pass & Beam & Inc. angle (deg) \\
\hline 20090603 & 154 & DSC & FQ02 & 22 \\
20090604 & 155 & ASC & FQ19 & 39 \\
20090607 & 158 & DSC & FQ19 & 39 \\
20090608 & 159 & ASC & FQ02 & 22 \\
20090610 & 161 & DSC & FQ06 & 26 \\
20090611 & 162 & ASC & FQ15 & 35 \\
20090617 & 168 & DSC & FQ10 & 30 \\
20090624 & 175 & DSC & FQ14 & 34 \\
20090701 & 182 & DSC & FQ19 & 39 \\
20090702 & 183 & ASC & FQ02 & 22 \\
20090704 & 185 & DSC & FQ06 & 26 \\
20090711 & 192 & DSC & FQ10 & 30 \\
20090712 & 193 & ASC & FQ11 & 31 \\
20090721 & 202 & DSC & FQ02 & 22 \\
20090722 & 203 & ASC & FQ19 & 39 \\
20090726 & 207 & ASC & FQ02 & 22 \\
20090804 & 216 & DSC & FQ10 & 30 \\
20090811 & 223 & DSC & FQ14 & 34 \\
20090812 & 224 & ASC & FQ06 & 26 \\
20090815 & 227 & ASC & FQ19 & 39 \\
20090818 & 230 & DSC & FQ19 & 39 \\
20090819 & 231 & ASC & FQ02 & 22 \\
20090822 & 234 & ASC & FQ15 & 35 \\
20090829 & 241 & ASC & FQ11 & 31 \\
\hline
\end{tabular}

would compensate the loss of temporal sensitivity, especially from the final application point of view. A complete analysis in this direction will be carried out in the future.

\section{B. PolSAR data pre-processing}

A pre-processing block is applied to all selected images in order to use the proposed methodology. It consists basically of three steps. The first one is the multi-look or speckle filtering (using a simple box car of $9 \times 9$ pixels), which produces the estimates of the polarimetric covariance matrix at each pixel. The second step consists in the geocoding of all data over the same geographical grid. Thus, all the resulting images are in the same reference grid, regardless of their beam or track. The third step involves the extraction of the polarimetric observables employed in the PCA. A total of 16 polarimetric parameters, listed at Table III, are employed in the analysis. As previously anticipated, in this example the entries of the covariance matrix are merged with entries from the polarimetric coherency matrix and outputs of its eigenvector decomposition. 

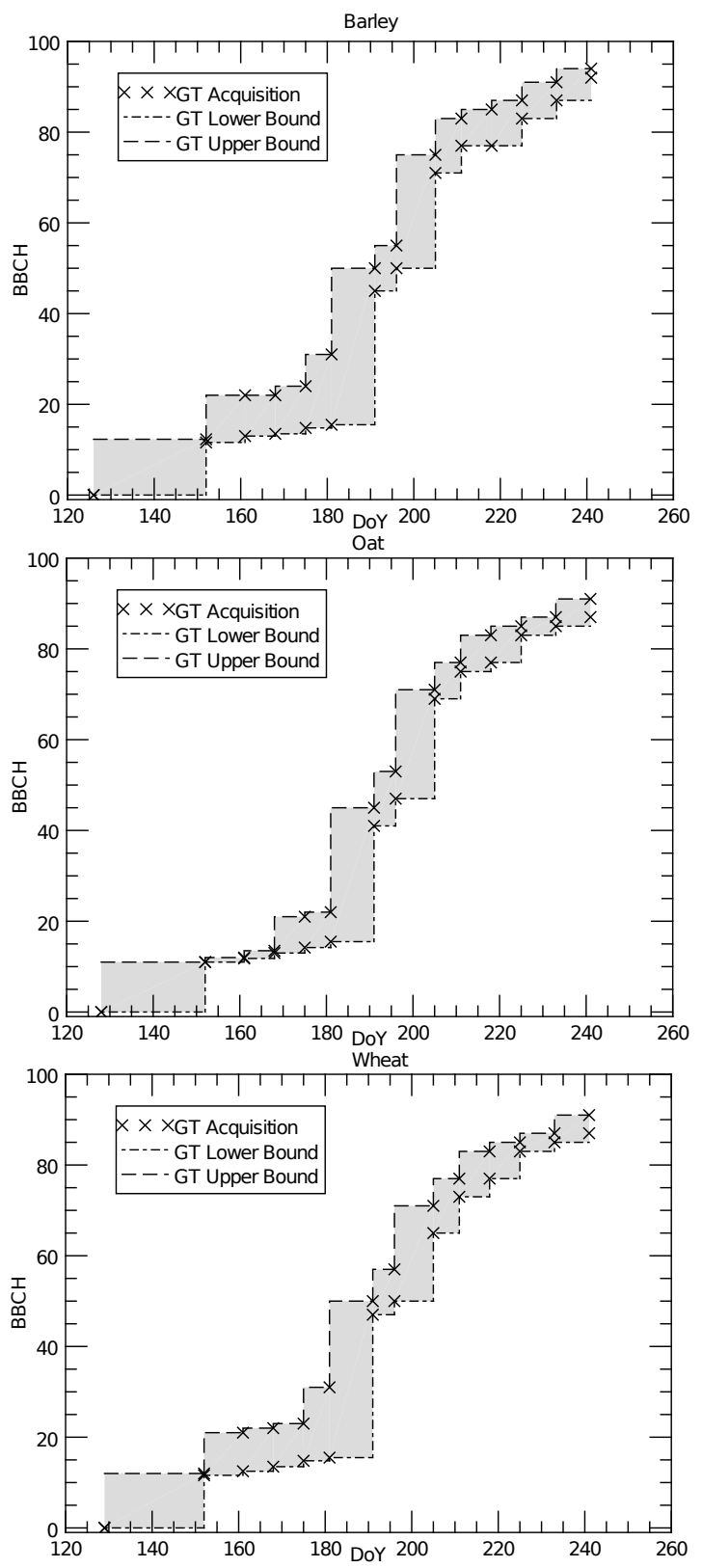

Fig. 5. Available phenology for each monitored crop: barley (top), oat(middle) and wheat (bottom). Cross marks denote the provided ground data (GT), and the shadowed region denotes the interpolated values. In each case the first phenological value corresponds to the sowing date.

\section{Model generation}

For simplicity, the model generation is carried out using the polarimetric covariance matrices averaged at parcel level. As introduced in Section II-B, the automatic extraction of the characteristics is performed by means of a PCA approach. Then, for comparison purposes, models have been generated for each crop independently and also combining the data of different crop types. The distribution of the coefficients of each polarimetric observable employed in each component, resulting from the PCA, is shown in Fig. 6. Each coefficient is directly related to the weight of each observable in the resulting dimension of the reprojected space. In all cases, the cumulative variance reached with three components is above $70 \%$ from the original set of sixteen observables, thus the loss of information is quite low when a 3-dimensional state space is used.

In order to improve the statistical analysis, increasing the number of significant samples, the PCA space definition has been also carried out by using combinations of different crop types, since they share actually similar biophysical features, hence representing with a common model the evolution of more than one crop. The first combination consists of only wheat and barley, 
TABLE III. LIST OF POLARIMETRIC OBSERVABLES CONSIDERED AT EACH ACQUISITION.

$\begin{array}{cc}\text { Polarimetric observable } & \text { Description } \\ |H H|^{2} & \text { Backscattering coefficient in the co-polar horizontal channel } \\ |V V|^{2} & \text { Backscattering coefficient in the co-polar vertical channel } \\ |H V|^{2} & \text { Backscattering coefficient in the cross-polar channel } \\ \left|P_{1}\right|^{2} & \text { Backscattering coefficient in the 1st Pauli channel }\left(|H H+V V|^{2} / 2\right) \\ \left|P_{2}\right|^{2} & \text { Backscattering coefficient in the 2nd Pauli channel }\left(|H H-V V|^{2} / 2\right) \\ \rho_{H H V V} H & \text { Correlation magnitude between co-polar channels } \\ \phi_{H H V V} H & \text { Polarimetric phase between co-polar channels } \\ \rho_{H H H V} H & \text { Correlation magnitude between horizontal co-polar and cross-polar channels } \\ \phi_{H H H V} H & \text { Polarimetric phase between horizontal co-polar and cross-polar channels } \\ \rho_{V V H V} & \text { Correlation magnitude between vertical co-polar and cross-polar channels } \\ \phi_{V V H V} & \text { Polarimetric phase between horizontal co-polar and cross-polar channels } \\ \rho_{P_{1} P_{2}} & \text { Correlation magnitude between 1st and 2nd Pauli channels } \\ \phi_{P_{1} P_{2}} & \text { Polarimetric phase between vertical co-polar and cross-polar channels } \\ H & \text { Entropy } \\ A & \text { Anisotropy } \\ \alpha_{1} & \text { Dominant alpha angle in the eigen-decomposition of T }\end{array}$
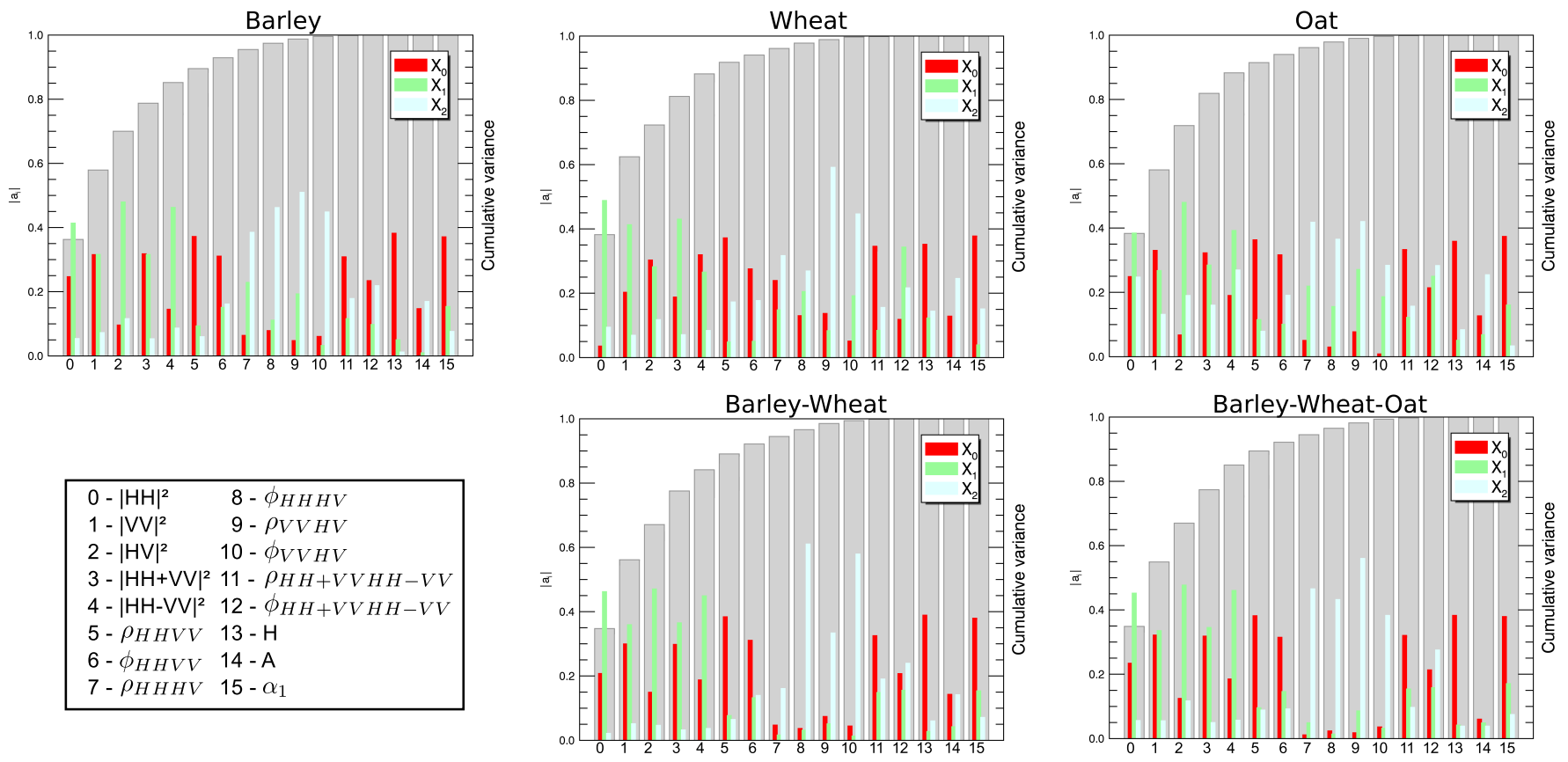

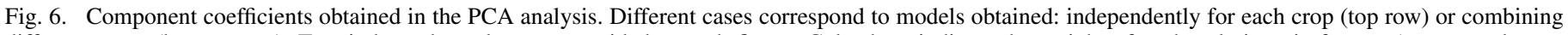

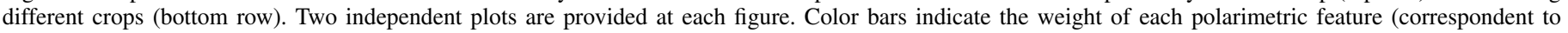

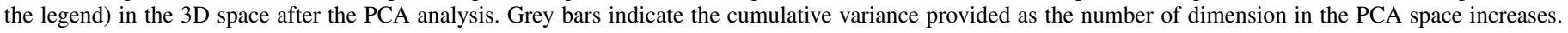


which are the most similar cereals in this study. In that case, the analysis is carried out by considering a total of five parcels. The last combination to be tested is formed by all the three crop types, using the whole set of parcels in the analysis. In this case the main behavior is similar to the one already described.

The 3D evolution models generated by the proposed automatic extraction technique are shown in Fig. 7 and 8 . The interpretation of the crop signatures is not straightforward, but some comments can be done to explain their basic meaning. The models in which barley is involved exhibit three easily recognizable regions: the first one between stages 0 and 4 (BBCH $00-39$ ), the second one between stages 5 and 6 (BBCH $40-69$ ), and the last one from stage 7 to 8 (BBCH 70 - 99). Not incidentally, this matches almost perfectly with a phenological scale with three main levels: vegetative (BBCH 00-49), reproductive (50-69) and maturation (BBCH 70-99) [26]. The behavior of the model along the first axis is defined in two zones: a linear increment from stages 0 to 6 and a saturation effect dominating both final stages 7 and 8 . In the second axis there are three clear zones: a decrease for stages 0 to 3 , an almost constant region for stages 4 to 6 , and an increase for the final stages. Although with different signs, a similar behavior is observed for the third dimension, for which an increase dominates from stages 0 to 4 , then a turning point occurs, and the central and last stages are defined by two different decrease ratios.

Even though the distributions of coefficients are similar to the other cases, the model obtained independently for wheat seems more chaotic and no direct interpretation can be found. The case for the oat model is also different, apart from a saturation effect in the third component during the last stages. In both cases (wheat and oat) there are only two parcels involved in the model generation, so the lower number of representative data is expected to produce less reliable models.

Although falling partially outside the scope of this work, it is also interesting to test the possibility of defining the three components of the evolution model in a manual way, that is, taken directly from the set of 16 polarimetric observables. Attending to the analysis shown in [27], three good candidates are the backscattering coefficient of the 2nd Pauli channel ( $\left.x_{0}\right)$, the dominant alpha angle $\left(x_{1}\right)$, and the correlation magnitude between the first two Pauli channels $\left(x_{2}\right)$. In this case these three polarimetric observables define directly the state space of the model. The phenology retrieval procedure proposed in this work will be also applied to this direct model and the results will be discussed in next Section. The physical interpretation of this model is relatively straightforward as each component represents only one observable. Although the signatures are different depending on the crop type, the following considerations hold for all of them. There is an increase at both early stages and the late ones in the $x_{0}$ component, driven by the initial growth of the plants and the increase of their randomness at the last dates, respectively. In $x_{1}$ there is also an increase during the whole cycle but for the two last stages, when its value saturates, showing an increasing departure from the surface scattering produced by the ground. The same behavior serves to describe the $x_{2}$ component, and thus the signature in the plane defined by the last two coordinates describes an almost linear trend. Nevertheless, the manual definition of the state-space model has the main drawback of being less flexible, needing a polarimetric analysis prior the definition.

\section{Estimation results}

In this Section the results that have been obtained employing the proposed methodology are presented and discussed. The estimation of phenology is carried out using the previously generated models and the available polarimetric acquisitions, considered as input data. The results obtained with the model derived independently for each crop type are shown in Fig. 9 and 10. The estimations are performed at pixel level, i.e. an estimate is provided for each pixel of the multilooked and geocoded images. Fig. 9 compares the obtained results with the ground data for the three crop types. The estimation range (min-max) at pixel level is denoted by the whiskers. In accordance with the number of available parcels, a total of three estimation ranges are shown for barley and only two for wheat and oat. In general, an increase in the variance of the estimates as a function of date is observed for all crop types, which is not present in the ground data. Moreover, a slight underestimation is observed in the second half of the cultivation cycle, which is larger in the case of oat fields. The temporal variation of the phenological estimates is quite smooth as observed shown in Fig. 10. However, within each field we can observe a noticeable heterogeneity in the phenology estimates. Such an heterogeneity maybe induced by the natural variability of the crops (some areas develop faster than others), as well as by the intrinsic variance in the radar data. It must be stated that we have not applied a segmentation or similar image processing technique to the data, which would contribute to get more spatially homogeneous estimates. Such a processing is left for future work.

The validation of the obtained estimates against the ground truth has been carried out as in a classification case, since phenological intervals can be regarded as different classes of the same crop. For each evaluated case, a confusion matrix and the $\kappa$ coefficient [29] are provided. The evaluation is established over 6 phenological intervals (i.e. classes) which are defined in Table IV. It should be remarked here that the estimation is obtained in a continuous phenological BBCH scale, but a discretization is applied later in order to compare with the provided ground truth. The mode (most frequent value) of the pixel estimates within each parcel is used for comparison with the ground data. For this comparison, also the ground truth data have to be adapted, so the mean value of the provided ranges is employed in the analysis.

Using the models generated independently for each crop type, we obtained the classification results shown in Table V. For both barley and wheat a significant $\kappa$ value is obtained, despite a lack of accuracy is exhibited during the intermediate stages. In all crop types, the highest accuracy in the estimation is obtained for the first and last phenological stages. In the case of barley and wheat crops, some accuracy is also achieved during the vegetative phase. However, the accuracy in the reproductive and in 

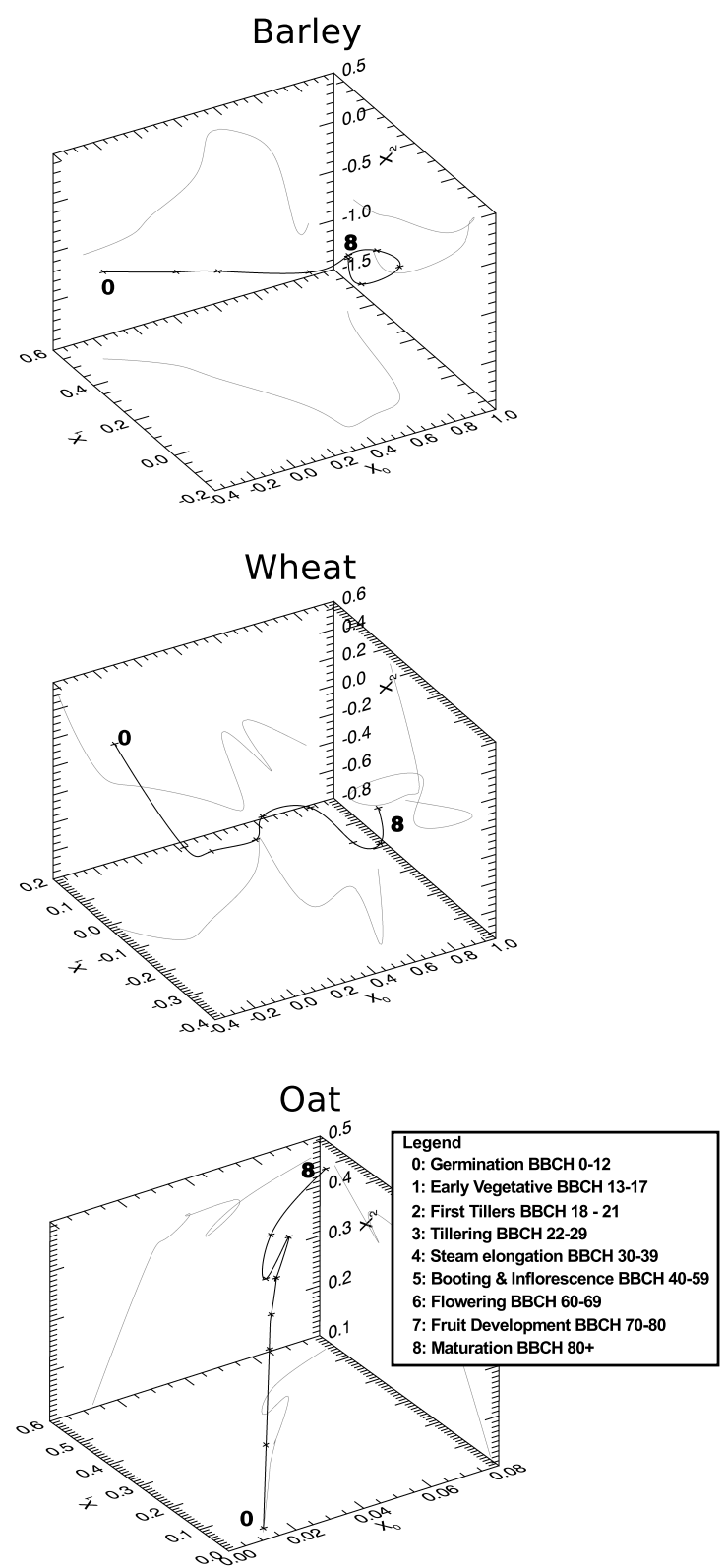

Fig. 7. Evolution model obtained by PCA for the three crops analyzed independently. Each cross mark represents the cluster center in the state-space model, with its corresponding phenological interval.

the first part of the maturation phase is low. Observing Fig. 9, it seems that the nonlinear behavior of phenology as a function of time (or date) is not followed properly by the estimation procedure. Hence, other potential filtering approaches (different from EKF) could be tested to avoid this problem. Anyway, the general accuracy in both crop types is high enough, considering the different uncertainty sources involving the ground data. In contrast, in oat fields there is a lack of accuracy in all the intermediate stages and thus the general estimation accuracy is lower.

If the model generated jointly for both barley and wheat is considered, the accuracy during the intermediate stages is significantly improved, as shown in Table VI. The overall performances of the estimation can be considered almost the same as the particular field application (independent models for each crop type) having a total accuracy of $76 \%$ and a $\kappa$ value of 0.69 . In this case the accuracy in the estimation of the intermediate stages is more uniform, showing values above $55 \%$ in all user and producer accuracies but in one case. This can be explained due to the fact that in this scenario the model is created with 

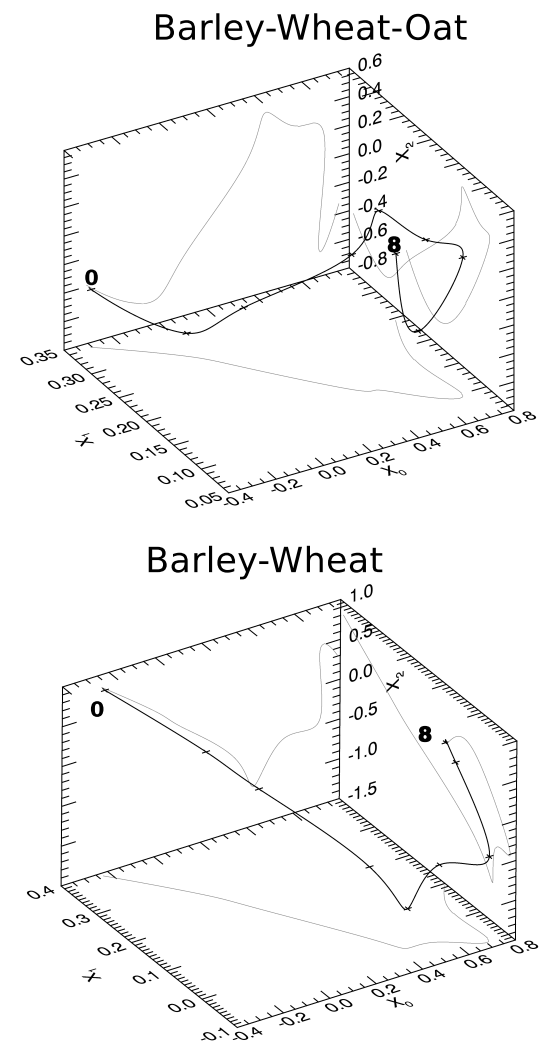

Fig. 8. Evolution model obtained by PCA for two combinations of crops. Each cross mark represents the cluster center in the state-space model, with its corresponding phenological interval.
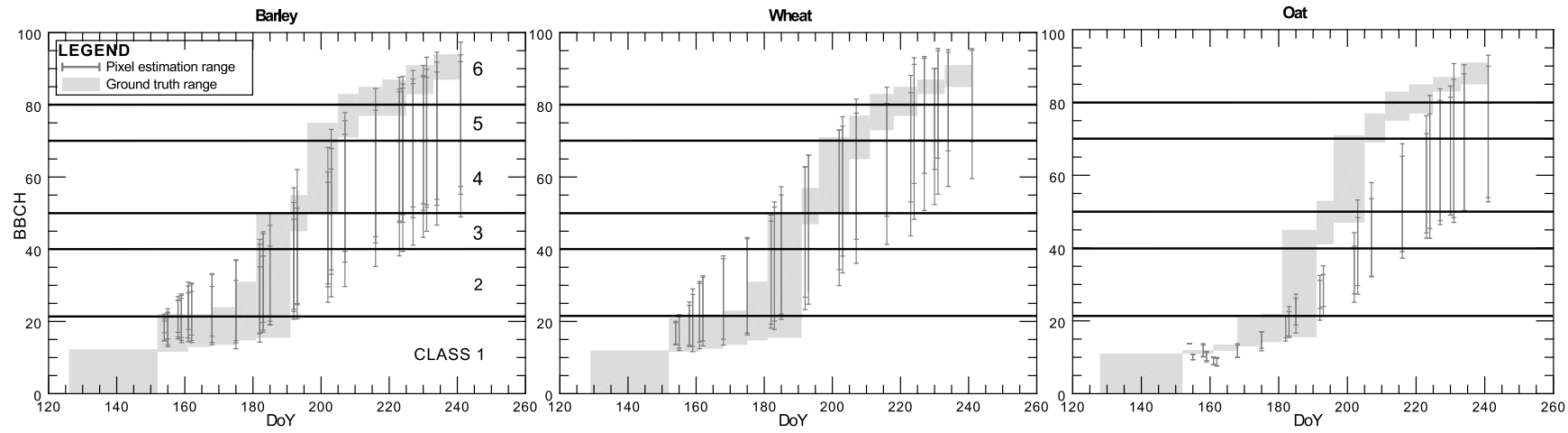

Fig. 9. Statistics of the phenology estimates obtained for the three crop types following the proposed methodology: barley (left), wheat (middle) and oat (right).

more significant samples and thus the estimation is performed with a robust methodology.

For completeness, the results obtained employing the polarimetric observables directly as components of the state-space model are shown in Table VII. In this case, the general accuracy does not increase, and the lack of sensitivity is even higher for later stages (3 to 5$)$.

Although the number of parcels available to perform the analysis is low, the results obtained for wheat and barley show clearly the potential of the methodology. Moreover, the strategy of combining parcels to obtain a more significant model has proven to be useful, based on an increase in the number of samples included in the learning stage. It should be reminded that different beams are included in the data in order to provide a fine temporal sampling in both model generation and variable estimation, so it is likely that this incidence angle combination is introducing some level of uncertainty at both stages (modeling and estimation). The available ground truth produces also some level of uncertainty. In Fig. 5 only the minimum and maximum 
TABLE IV. CLASSES DEFINITION IN THE CONFUSION MATRIX ANALYSIS.

\begin{tabular}{ccc} 
Class & BBCH Range & Description \\
\hline 1 & $00-21$ & \\
2 & $22-39$ & Vegetative \\
3 & $40-49$ & \\
\hline 4 & $50-69$ & Reproductive \\
\hline 5 & $70-79$ & Maturation \\
6 & $80-99$ & \\
\hline
\end{tabular}

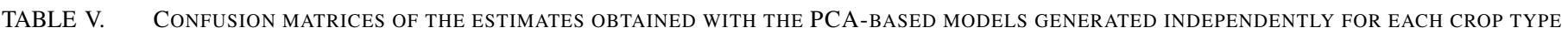

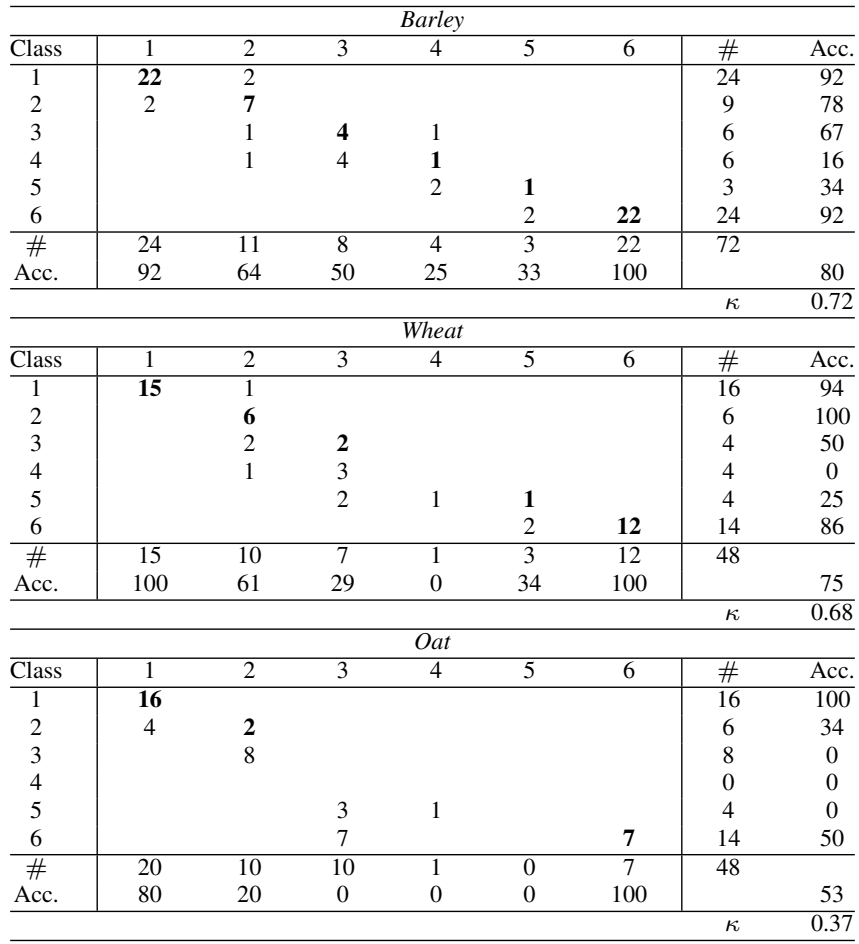

TABLE VI. CONFUSION MATRIX OF THE ESTIMATES OBTAINED WITH THE PCA-BASED MODEL GENERATED JOINTLY WITH BARLEY AND WHEAT

\begin{tabular}{c|cccccc|cc}
\hline Class & 1 & 2 & 3 & 4 & 5 & 6 & $\#$ & Acc. \\
\hline 1 & $\mathbf{3 5}$ & 5 & & & & & 40 & 87 \\
2 & & $\mathbf{1 4}$ & 1 & & & & 15 & 93 \\
3 & & 3 & $\mathbf{7}$ & & & & 10 & 70 \\
4 & & 2 & 2 & $\mathbf{6}$ & & & 10 & 60 \\
5 & & & 2 & 1 & $\mathbf{4}$ & & 7 & 57 \\
6 & & & & 1 & 12 & $\mathbf{2 5}$ & 38 & 66 \\
\hline$\#$ & 35 & 24 & 12 & 8 & 16 & 25 & 120 & \\
Acc. & 100 & 58 & 58 & 75 & 25 & 100 & & 76 \\
\hline
\end{tabular}

TABLE VII. CONFUSION MATRIX OF THE ESTIMATES OBTAINED WITH THE MODEL GENERATED WITH DIRECT POLARIMETRIC OBSERVABLES AND JOINTLY FOR BARLEY AND WHEAT

\begin{tabular}{c|cccccc|cc}
\hline Class & 1 & 2 & 3 & 4 & 5 & 6 & $\#$ & Acc. \\
\hline 1 & $\mathbf{4 0}$ & & & & & & 40 & 100 \\
2 & 2 & $\mathbf{1 3}$ & & & & & 15 & 87 \\
3 & & 5 & $\mathbf{5}$ & & & & 10 & 50 \\
4 & & & 10 & & & & 10 & 0 \\
5 & & & 5 & 2 & & & 7 & 0 \\
6 & & 1 & 20 & 1 & 2 & $\mathbf{1 4}$ & 38 & 37 \\
\hline$\#$ & 42 & 19 & 40 & 3 & 2 & 14 & 120 & \\
Acc. & 95 & 68 & 12 & 0 & 0 & 100 & & 60 \\
\hline \multicolumn{1}{l}{} & & & & & & & $\kappa$ & 0.50
\end{tabular}




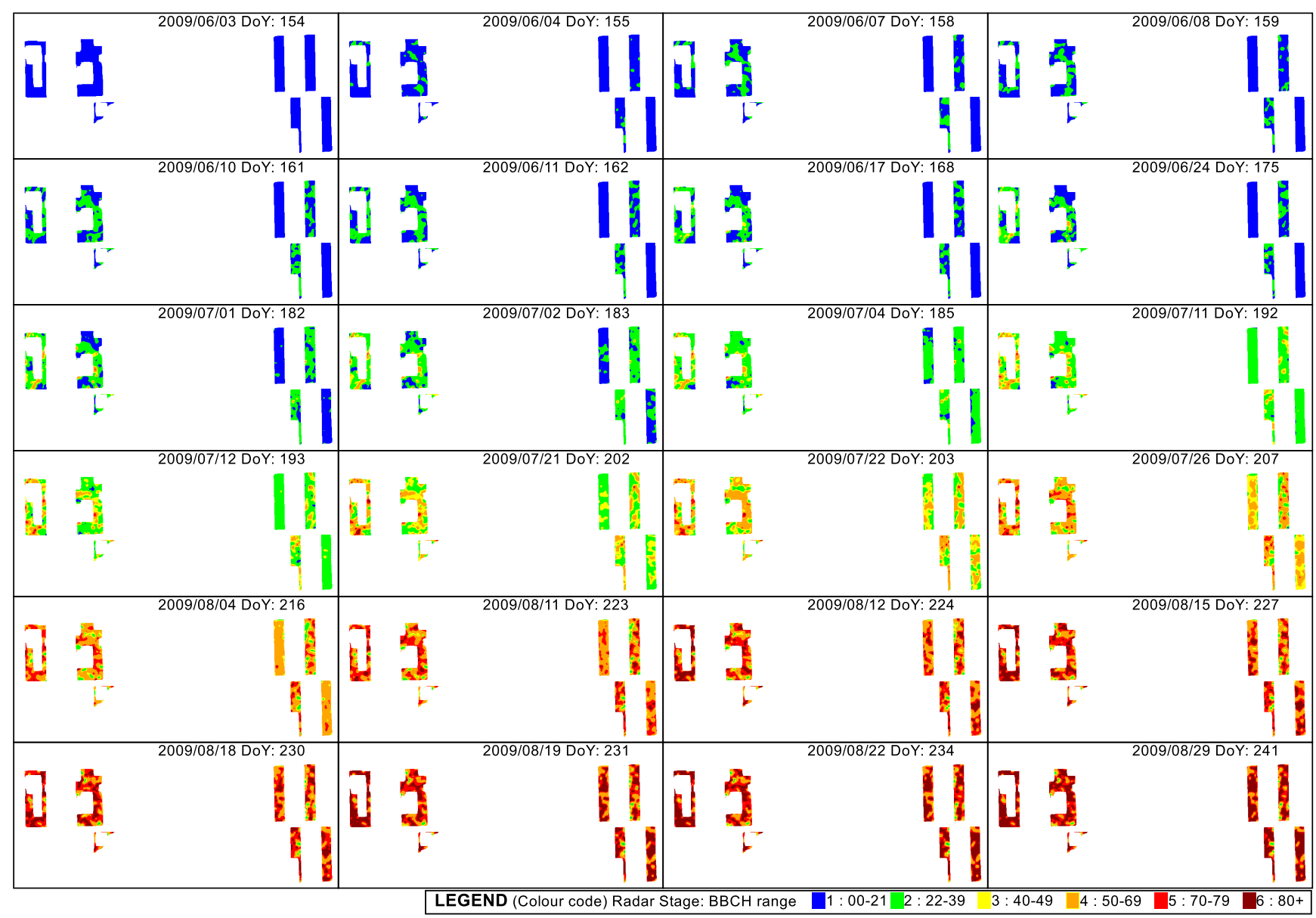

Fig. 10. Mosaic of all maps of retrieved phenology at pixel level using the proposed methodology.

phenological values at each date are depicted, but for some dates the supplied values are quite heterogeneous. For instance, in the case of barley two or three different phenological ranges, later assigned to different clusters, are provided for the same date in the vegetative phases, but there is no information about their spatial distribution or the percentage of areas under each range. To avoid stronger assumptions, a uniform distribution is considered within the provided minimum and maximum range. However, the real scenario could differ much from this hypothesis.

At comparative level, oat results are less accurate than those obtained for wheat and oat. It can be explained in part because of a lower sensitivity from the radar response to this crop, as pointed out in [27]. In order to obtain more reliable results, it seems that in this case a larger number of data is still needed. The higher radar sensitivity for wheat and barley explains the better performance with the same number of parcels. Either increasing the parcels in the modeling stage or the revisit time could affect positively the quality of the obtained estimates.

To provide an extended qualitative assessment, the estimation procedure has been applied also to a bigger area where the only available ground truth information is the crop type. It consist in the surrounding area to the reference set, and the distribution of parcels and crop types is illustrated at Fig. 11. A total set of 59 fields are considered. In particular, the set is divided in 10 barley, 8 oat and 41 spring wheat fields.

Exploiting the model generated for the reference set of parcels, the estimation is applied for each depicted field exploiting the same stack of Radarsat-2 images. The obtained results, shown in Fig. 12, are plotted against the provided ground truth for the reference set of parcels. In general, there exist a common trend in the three set of crop types following the reference ground truth. It can be observed that, also for this evaluation, oat fields present the highest deviation from the reference ground truth. In Fig. 13 maps of the estimates are presented for five different dates. Although there is no knowledge of the actual phenological stages at any date, it is expected that the fields in the area behave in a similar way. For that reason with this evaluation we can not assess quantitatively the methodology but prove visually that the methodology is useful and exportable.

Working in a reduced state space of three dimensions has the advantage of providing an easy interpretation, since the evolution can be represented graphically. However, there is no restriction in the use of a higher dimensional space. The same methodology 


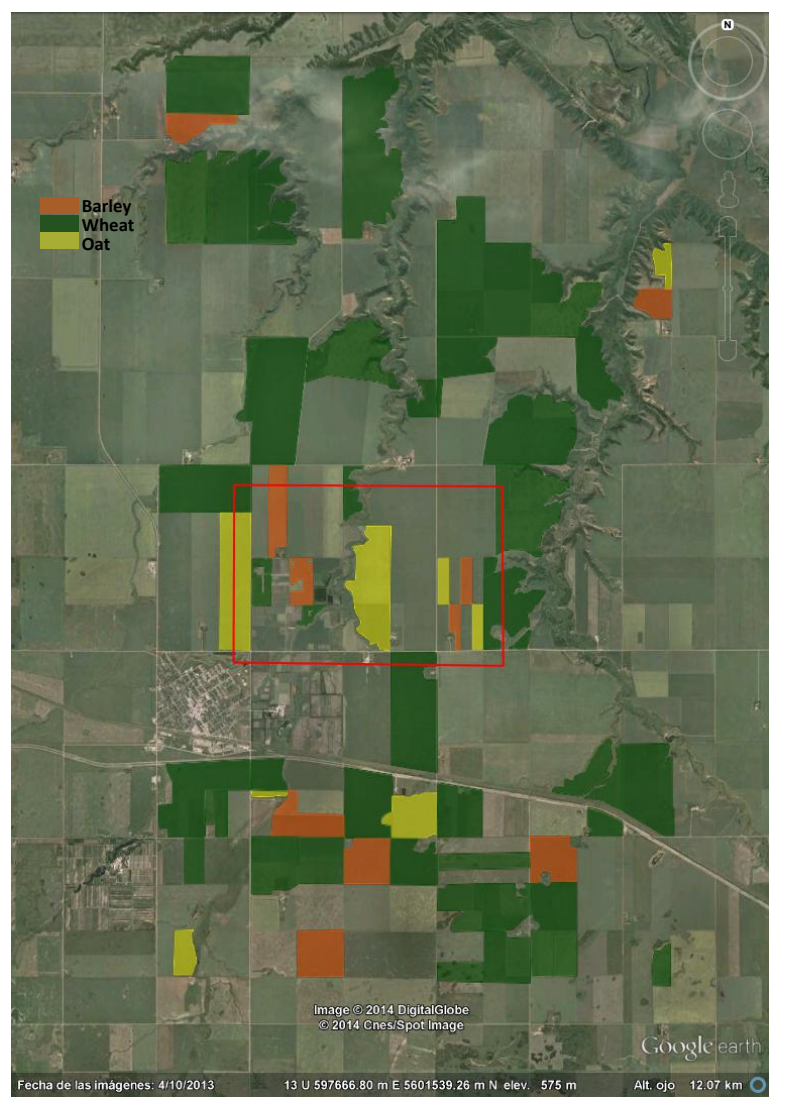

Fig. 11. Distribution of cereal fields over the whole area where the phenological stage is retrieved. The red box indicates the location of the reference set of parcels. Photo courtesy of Google Earth.
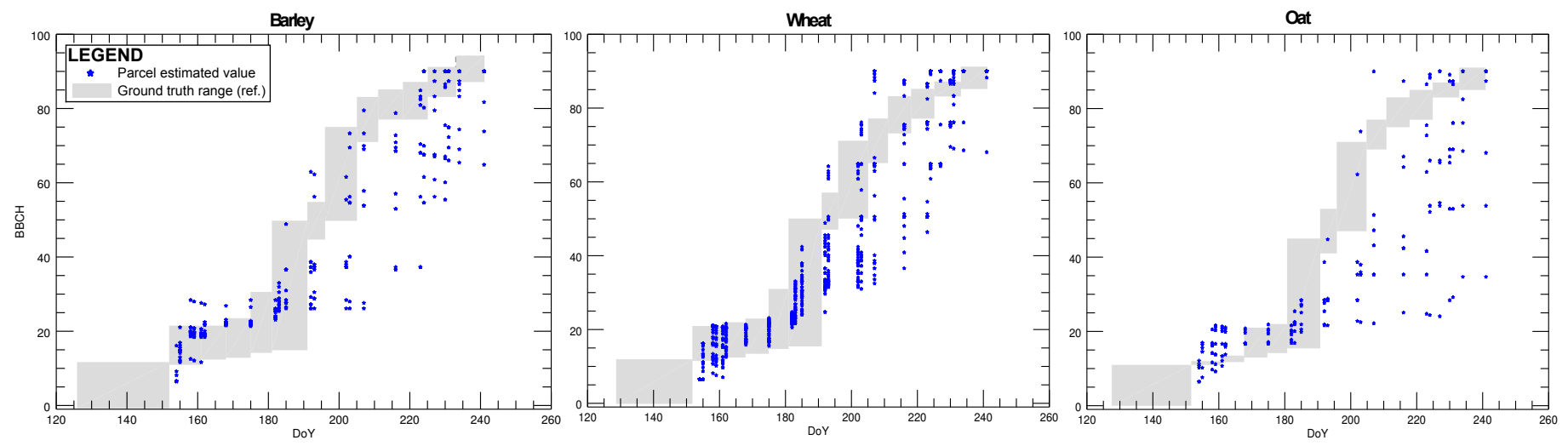

Fig. 12. Estimated phenological stage for each crop type over the extended set of fields. The ground truth for the learning set of parcels is provided for reference. 


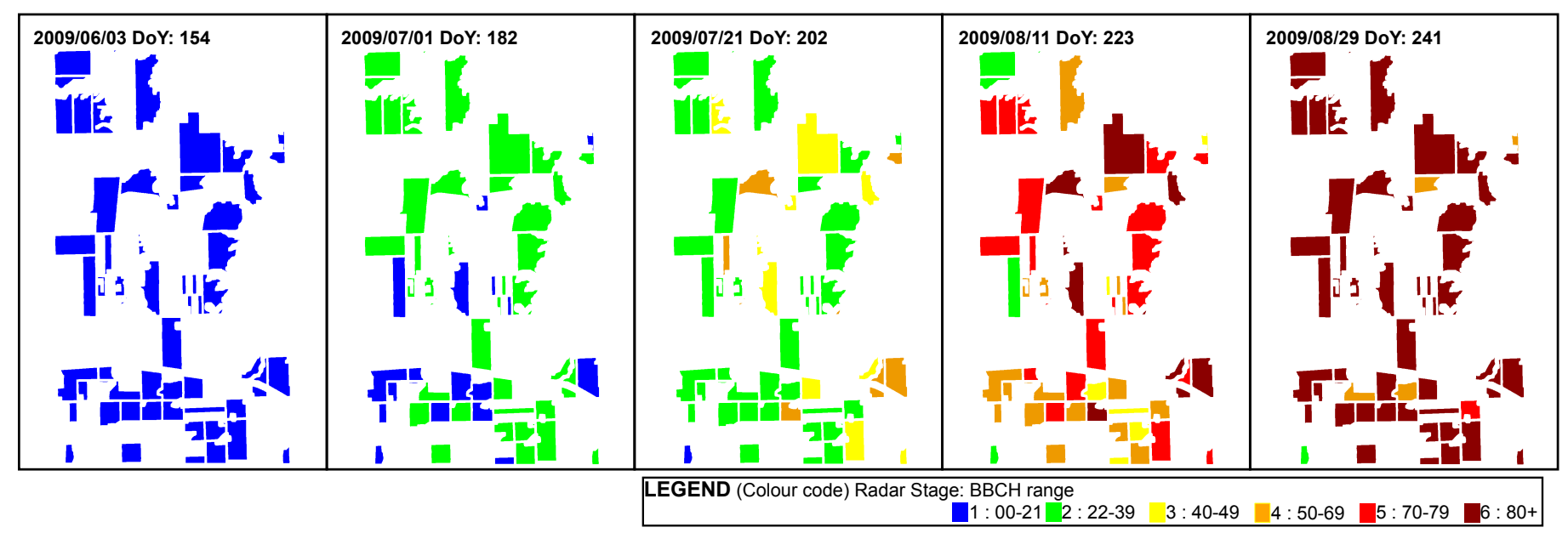

Fig. 13. Temporal subset corresponding to a sequence of the estimated phenological stages over the extended area.

is suitable for a $\mathrm{N}$-dimensional space. In the analyzed scenario, for instance, cumulative variances close to $90 \%$ can be reached considering 5 dimensions, so it has to be tested how, despite the reduced number of data, a higher dimension approximation affects the estimation.

Regarding the results obtained with a manual selection of the polarimetric observables that define the state space, two main ideas can be extracted. The first one is related with the accuracy obtained with the methodology. The direct usage of the polarimetric observables as components in the methodology has provided less accurate estimates than the ones obtained with the PCA. Different combinations of parameters could be employed to maximize the estimation accuracy. However, with the automatic analysis this is not necessary. The second idea is involved directly with the potential of the approach to be exported to other fields or crop types. In the direct selection of components, a manual assessment has to be carried out externally to apply the methodology, i.e. to detect the most sensitive parameters to phenology. This has to be repeated each time a new crop type is going to be monitored. Alternatively, an automatic analysis tool, as the one proposed in this paper, provides the assessment in a more effective way.

\section{CONCLUSION}

In this work a novel approach to analyze and monitor with remote sensing the evolution of crop fields has been proposed and tested. The methodology is based on considering the crop evolution as a dynamic system, hence providing a real-time monitoring based on remote sensing input data. It has been shown that multitemporal data are suitable to define an approach in that context. Although the methodology has been tested using polarimetric SAR data, the approach is fully capable to define the same strategy by employing any other remotely sensed data with sensitivity to different biological crop variables. The results obtained, based on a subset of parcels for which ground truth is available and extended to a surrounding area of parcels of unknown ground truth, have shown the potential of the presented framework. The real-time capabilities of this approach enable the final users with the possibility to obtain estimates as remote sensing data are available, and hence contribute to develop new practical applications. For instance, real-time alerts about crop anomalies can be easily derived from this methodology. In addition, the prediction stage could be used standalone to infer future stages in the phenological evolution, from a current known one, or the dates in which a particular crop will reach some predefined condition. Such tools would help to increase crop yields and to optimize the available farming resources.

Besides final applications, the presented approach has a great potential in this field thanks to its flexibility and its the capability to be exported to different scenarios. The definition of the methodology in the state-space domain enables the use of different filtering techniques exploiting the temporal dimension. The entire procedure defines a close optimal approach for the phenological estimation, despite nonlinear behaviors. In addition to different types of input/output data, the proposed methodology represents a general algorithm with a modular structure, as shown in Fig. 1. In this sense, this work sets up the basis for a dynamical approach focused on crop monitoring. As a proof of concept, in this study a PCA/EKF combination has been employed, but alternative modeling stages (e.g. ANN or SVM) and filtering strategies (e.g. particle filters or grid-based estimators) can be applied. In particular, filtering strategies specific for nonlinear scenarios would produce a better performance in the estimation stage. A similar strategy was employed successfully in [30], [31] for interferometric phase unwrapping. In these works, the dynamics definition of the phase unwrapping problem in the state-space domain allowed the exploitation of different filtering approaches, as the grid-based and the particle filter. 
The proposed model generation from empirical data, employing an automatic analysis tool, has proven to be successful in this context. The reduction from a large number of variables can be done using the PCA and without any supervision. Only the cluster ranges for the model have to be specified by the user in order to create the model. Also the proposed numerical linearization has been effective, so the overall idea of the EKF has been successfully translated and applied to the dynamic phenological evolution problem.

From the analyzed dataset, satisfactory results are obtained for wheat and barley with an agreement close to $\kappa=0.7$ during the whole cultivation cycle. In the case of oat the results showed only good sensitivity during the first and last stages of the phenological evolution. In all cases, the number of available fields is rather low. Consequently, the main limitations in this work stem from the low number of fields involved in the study. Naturally, this could be solved using more fields to get the most representative features for each crop within the model. In this regard, an online modeling methodology could be defined in the future. This approach will increase the model accuracy each time an estimation is performed. Moreover, a further analysis should also be driven to assess the effects of the incidence angle in a common scenario and how changes on the temporal sampling rate (considering different stacks of images) affect the dynamic process underneath.

Together with other sources of remote sensing data, the methodology is suitable for different polarimetric modes. A further analysis in this matter will be made with the objective of determining the impact of the polarimetric configuration and how the dimensional reduction affects the phenological estimation quality compared to those obtained for the full polarization case. Moreover, the adopted filtering strategy allows us to further establish the mechanisms to fuse data from different remote sensing sources, for instance SAR and hyperspectral data. This scenario would increase the temporal resolution, due to different sampling rates and the sensitivity to phenology, being each of the sensor types responsive to different aspects of the crop field evolution.

\section{ACKNOWLEDGMENT}

Radarsat-2 Data and Products (C)MacDonald, Dettwiler and Associates Ltd. (MDA, 2009) - All Rights Reserved. Radarsat is an official trademark of the Canadian Space Agency (CSA). All Radarsat-2 images have been provided by MDA and CSA in the framework of the ESA funded AgriSAR2009 campaign. The ground data collection was conducted by the Indian Head Agriculture Research Facility (IHARF) and the University of Regina.

\section{REFERENCES}

[1] M. S. Moran, Y. Inoue, and E. M. Barnes, "Opportunities and limitations for image-based remote sensing in precision crop management," Remote Sensing of Environment, vol. 61, pp. 319-346, 1997.

[2] P. J. Pinter Jr. et al., "Remote sensing for crop management," Photogrammetric Engineering and Remote Sensing, vol. 69, no. 6, pp. 647-664, June 2003.

[3] T. Sakamoto, B.D. Wardlow, and A.A. Gitelson, "Detecting spatiotemporal changes of corn developmental stages in the U.S. corn belt using MODIS WDRVI data," Geoscience and Remote Sensing, IEEE Transactions on, vol. 49, no. 6, pp. 1926-1936, June 2011.

[4] H. McNairn and B. Brisco, "The application of C-band polarimetric SAR for agriculture: a review," Can. J. Remote Sensing, vol. 30, no. 3, pp. 525-542, 2004.

[5] A. Bouvet, T. Le Toan, and N. Lam-Dao, "Monitoring of the rice cropping system in the Mekong delta using ENVISAT/ASAR dual polarization data," Geoscience and Remote Sensing, IEEE Transactions on, vol. 47, pp. 517-526, 2009.

[6] J. M. Lopez-Sanchez and J. D. Ballester-Berman, "Potentials of polarimetric SAR interferometry for agriculture monitoring," Radio Science, vol. 44, RS2010, March 2009.

[7] N. Poole, "Disease management and crop canopies," Tech. Rep., Grains Research and Development Corporation, 2009.

[8] R. Zurita-Milla, J. A. E. van Gijsel, N. A. S. Hamm, P. W. M. Augustijn, and A. Vrieling, "Exploring spatiotemporal phenological patterns and trajectories using self-organizing maps," Geoscience and Remote Sensing, IEEE Transactions on, vol. 51, no. 4, pp. 1914-1921, April 2013.

[9] M. Törmä, S. Anttila, S. Hatunen, E. Järvenpää, and M. Kervinen, "Vegetation phenological features from daily modis NDVI time series," in Proceedings of the ESA Living Planet Symposium, vol. ESA-SP 722, Edinburgh, U. K., September 2013.

[10] F. Vicente-Guijalba, T. Martinez-Marin, and J. M. Lopez-Sanchez, "Crop phenology estimation using a multitemporal model and a kalman filtering strategy," Geoscience and Remote Sensing Letters, IEEE, vol. 11, no. 6, pp. 1081-1085, June 2014.

[11] V. R. N. Pauwels, A. Balenzano, G. Satalino, H. Skriver, N. E. C. Verhoest, and F. Mattia, "Optimization of soil hydraulic model parameters using synthetic aperture radar data: An integrated multidisciplinary approach," Geoscience and Remote Sensing, IEEE Transactions on, vol. 47, no. 2, pp. 455-467, Feb 2009.

[12] W. Kleynhans, J. C. Olivier, K. J. Wessels, B. P. Salmon, F. Van den Bergh, and K. Steenkamp, "Detecting land cover change using an extended kalman filter on MODIS NDVI time-series data," Geoscience and Remote Sensing Letters, IEEE, vol. 8, no. 3, pp. 507-511, May 2011.

[13] J. M. Lopez-Sanchez, S. R. Cloude, and J. D. Ballester, "Rice phenology monitoring by means of SAR polarimetry at X-band," Geoscience and Remote Sensing, IEEE Transactions on, vol. 50, no. 7, pp. 2695-2709, July 2012.

[14] J. M. Lopez-Sanchez, F. Vicente-Guijalba, J. D. Ballester-Berman, and S. R. Cloude, "Polarimetric response of rice fields at C-Band: Analysis and phenology retrieval," Geoscience and Remote Sensing, IEEE Transactions on, vol. 52, no. 5, pp. 2977-2993, May 2014.

[15] C. Rossi and E. Erten, "Paddy-rice monitoring using TanDEM-X," Geoscience and Remote Sensing, IEEE Transactions on, vol. 53, no. 2, pp. 900-910, Feb 2015.

[16] S. Haykin and L. Li, "Nonlinear adaptive prediction of nonstationary signals," Signal Processing, IEEE Transactions on, vol. 43, no. 2, pp. 526-535, FEB 1995. 
[17] S. Haykin, R. Bakker, and B.W. Currie, "Uncovering nonlinear dynamics-the case study of sea clutter," Proceedings of the IEEE, vol. 90, no. 5, pp. 860-881, May 2002.

[18] M. S. Grewal and A. P. Andrews, Kalman filtering: theory and practice using MATLAB, second edition, John Wiley \& Sons, 2001.

[19] R. E. Kalman, "A new approach to linear filtering and prediction problems," ASME - Journal of Basic Engineering, Transactions of the, vol. 82, pp. $35-45,1960$.

[20] R. E. Kalman and R. S. Bucy, "New results in linear filtering and prediction theory," ASME - Journal of Basic Engineering, Transactions of the, vol. 83, pp. 95-107, 1961.

[21] C. De Bernardis, F. Vicente-Guijalba, T. Martinez-Marin, and J. M. Lopez-Sanchez, "Estimation of key dates and stages in rice crops using PolSAR time series and a particle filtering approach," Journal of Selected Topics in Applied Earth Observations and Remote Sensing, 2014, Submitted.

[22] S. R. Cloude, Polarisation. Applications in remote sensing, Oxford University Press, 2009.

[23] J.-S. Lee and E. Pottier, Polarimetric radar imaging. From basics to applications, CRC Press, 2009.

[24] S. R. Cloude and E. Pottier, "An entropy based classification scheme for land applications of polarimetric SAR," Geoscience and Remote Sensing, IEEE Transactions on, vol. 35, no. 1, pp. 68-78, Jan 1997.

[25] J. C. Zadoks, T. T. Chang, and C. F. Konzak, "A decimal code for the growth stages of cereals," Weed Research, vol. 14, no. 6, pp. 415-421, 1974.

[26] U. Meier, "Growth stages of mono-and dicotyledonous plants (2nd ed.)," Tech. Rep., Federal Biological Research Centre for Agriculture and Forestry, 2001.

[27] J. M. Lopez-Sanchez, F. Vicente-Guijalba, J. D. Ballester-Berman, and S. R. Cloude, "Estimating phenology of agricultural crops from space," in Proceedings of the ESA Living Planet Symposium, vol. ESA-SP 722, Edinburgh, U. K., September 2013.

[28] R. Caves et al., “AgriSAR2009 Final Report, Vol. 1. Executive Summary, Data Acquisition, Data Simulation,” Tech. Rep., 2011, Available online: http://earth.esa.int/campaigns/.

[29] J. Cohen, "A coefficient of agreement for nominal scales," Educational and Psychological Measurement, vol. 20, no. 1, pp. 37-46, 1960.

[30] J. J. Martinez-Espla, T. Martinez-Marin, and J. M. Lopez-Sanchez, "Using a grid-based filter to solve InSAR phase unwrapping," Geoscience and Remote Sensing Letters, IEEE, vol. 5, no. 2, pp. 147-151, April 2008.

[31] J. J. Martinez-Espla, T. Martinez-Marin, and J. M. Lopez-Sanchez, "A particle filter approach for InSAR phase filtering and unwrapping," Geoscience and Remote Sensing, IEEE Transactions on, vol. 47, no. 4, pp. 1197-1211, April 2009.

\begin{tabular}{|c|}
\hline \\
\\
PLACE \\
PHOTO \\
HERE \\
\end{tabular}

Fernando Vicente-Guijalba was born in Elche, Alicante, Spain, in 1981. He received the Ingeniero Tecnico degree in telecommunication engineering and the Ingeniero degree in sound and image engineering from the University of Alicante in 2006 and 2011 respectively. He has been a Pre-Doctoral Fellow with the Signals, Systems and Telecommunications Groups, University of Alicante, since 2011. His current research interests include dynamical systems analysis with applications in the polarimetric and interferometric SAR methods.

PLACE

PHOTO

HERE
Tomas Martinez-Marin received the Tech. Eng. (B.S.) degree from the University of Alcala (UAH) in 1990, the M.S. and the Ph.D. degree in telecommunications engineering from the Technical University of Madrid (UPM) in 1995 and 1999, respectively. He joined the University of Alcala as Assistant Professor in 1990. In 1997, he joined the European University of Madrid (UEM) as Assistant Professor. Since 2000, he is with the Dept. of Physics, System Engineering and Signal Theory at the University of Alicante (UA), where he currently is Associate Professor. His research topics include reinforcement learning, optimal control, intelligent vehicles, and SAR filtering algorithms. 
PLACE

PHOTO

HERE
Juan M. Lopez-Sanchez (S'94, M'00, SM'05) was born in Alicante, Spain, in 1972. He received the Ingeniero and Doctor Ingeniero degrees in Telecommunication Engineering from the Technical University of Valencia (UPV), Valencia, Spain, in 1996 and 2000 , respectively. From 1998 to 1999 he worked as a predoctoral grantholder at the Space Applications Institute, Joint Research Centre of the European Commission, Ispra, Italy. Since 2000 he leads the Signals, Systems and Telecommunication Group of the University of Alicante, Spain, where he is full professor since November 2011.

His main research interests include microwave remote sensing for inversion of biophysical parameters, polarimetric and interferometric techniques, SAR imaging algorithms, and analytical and numerical models for multiple scattering problems.

In 2001, Dr. Lopez-Sanchez received the Indra Award for the best Ph.D. thesis about radar in Spain. From 2006 to 2012 he was the Chair of the Spanish Chapter of the IEEE Geoscience and Remote Sensing Society. He has coauthored more than 50 papers in refereed journals and more than 90 papers and presentations in international conferences and symposia. 